ARTICLE

Received 11 May 2016 | Accepted 20 Jan 2017 | Published 24 Mar $2017 \quad$ DOl: 10.1038/ncomms14659 OPEN

\title{
A chemical chaperone improves muscle function in mice with a RyR1 mutation
}

Chang Seok Lee ${ }^{1, \star}$, Amy D. Hanna ${ }^{1, \star}$, Hui Wang ${ }^{1}$, Adan Dagnino-Acosta ${ }^{1}$, Aditya D. Joshi ${ }^{1}$, Mark Knoblauch ${ }^{1}$

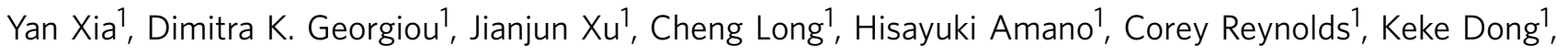
John C. Martin ${ }^{1}$, William R. Lagor ${ }^{1}$, George G. Rodney ${ }^{1}$, Ergun Sahin ${ }^{1}$, Caroline Sewry ${ }^{2}$ \& Susan L. Hamilton ${ }^{1}$

Mutations in the RYR1 gene cause severe myopathies. Mice with an 148951 mutation in the type 1 ryanodine receptor/ $\mathrm{Ca}^{2+}$ release channel (RyR1) display muscle weakness and atrophy, but the underlying mechanisms are unclear. Here we show that the 14895T mutation in RyR1 decreases the amplitude of the sarcoplasmic reticulum (SR) $\mathrm{Ca}^{2+}$ transient, resting cytosolic $\mathrm{Ca}^{2+}$ levels, muscle triadin content and calsequestrin (CSQ) localization to the junctional $S R$, and increases endoplasmic reticulum (ER) stress/unfolded protein response (UPR) and mitochondrial ROS production. Treatment of mice carrying the 14895T mutation with a chemical chaperone, sodium 4-phenylbutyrate (4PBA), reduces ER stress/UPR and improves muscle function, but does not restore SR $\mathrm{Ca}^{2+}$ transients in 14895T fibres to wild type levels, suggesting that decreased $\mathrm{SR} \mathrm{Ca}^{2+}$ release is not the major driver of the myopathy. These findings suggest that 4 PBA, an FDA-approved drug, has potential as a therapeutic intervention for RyR1 myopathies that are associated with ER stress.

\footnotetext{
${ }^{1}$ Department of Molecular Physiology and Biophysics, Baylor College of Medicine, One Baylor Plaza, Houston, Texas 77030, USA. ${ }^{2}$ Dubowitz Neuromuscular Centre, UCL Institute of Child Health and Great Ormond Street Hospital, 30 Guilford Street, London WC1N 1EH, UK. * These authors contributed equally to this work. Correspondence and requests for materials should be addressed to S.L.H. (email: susanh@bcm.tmc.edu).
} 


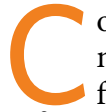
ore myopathies are the most common form of congenital myopathies and more than half of these myopathies arise from mutations in the gene (RYR1) for the skeletal muscle $\mathrm{Ca}^{2+}$ release channel or type 1 ryanodine receptor $(\mathrm{RyR} 1)^{1}$. $R Y R 1$ mutations in the human population occur at frequencies as high as 1 in 2000 (ref. 2). In mice some mutations in the Ryr1 gene increase SR $\mathrm{Ca}^{2+}$ leak $^{3}$ while others decrease $\mathrm{Ca}^{2+}$ permeation through RyR1 (ref. 4), raising the question of how mutations with opposing effects on both the amplitude of the $\mathrm{Ca}^{2+}$ transient and resting cytosolic $\mathrm{Ca}^{2+}$ concentrations produce similar disease symptoms. We have shown that a mutation in Ryrl that leads to the replacement of tyrosine with a serine at amino acid 524 in RyR1 (Y522S in humans, Y524S in mice), which cause both malignant hyperthermia and myopathy with cores, increases heat sensitivity, temperature-dependent SR $\mathrm{Ca}^{2+}$ leak, mitochondrial damage and oxidative/nitrosative stress $^{5}$. Despite substantial progress towards developing interventions for myopathies arising from $\mathrm{Ca}^{2+}$ leak in RyR1 (ref. 6), there are currently no FDA-approved drugs available for any RyR1 myopathy. The heat-induced life-threatening response in transgenic mice carrying the Y524S mutation is prevented by treatment with 5-aminoimidazole-4-carboxamide ribonucleoside ${ }^{7}$ and the myopathy is alleviated by anti-oxidants ${ }^{5}$. Since, as described in this manuscript, the IT mutation decreases RyR1 $\mathrm{Ca}^{2+}$ permeation and $\mathrm{Ca}^{2+}$ leak, it is unlikely that interventions that further decrease $\mathrm{Ca}^{2+}$ leak via RyR1 will be beneficial (and may even be harmful) in patients with mutations that either decrease $\mathrm{Ca}^{2+}$ permeation through RyR1 or affect its interaction with SR-lumenal proteins.

To develop interventions for RyR1 myopathies arising from RyR1 mutations that do not produce $\mathrm{Ca}^{2+}$ leak, we created mice with the I4895T mutation (equivalent to I4898T in humans). This mutation, identified initially by Lynch et al. ${ }^{8}$, is one of the most common RyR1 mutations in humans and has a variable presentation and penetrance ranging from myopathies such as Central Core Disease, which can present extremely mild to severe muscle impairment in a single family ${ }^{9-11}$ to a lethal early onset congenital core-rod myopathy ${ }^{12}$. Mice with the I4895T mutation on a different genetic background ${ }^{13}$ display a highly variable phenotype ranging from a severe muscle phenotype that dramatically increases in severity with age to a mild, nonprogressive myopathy. The ultrastructural changes detected in these mice were found to be consistent with premature muscle ageing ${ }^{14}$. However, for the most part, RyR1 myopathies are early onset and non-progressive or slowly progressive ${ }^{15}$ suggesting that the subset of I4895T mice with the severe, age-dependent phenotype are not truly representative of human early onset, slowly progressive RyR1 myopathy. In the present study we demonstrate that persistent endoplasmic reticulum (ER) stress/UPR and excessive mitochondrial reactive oxygen species (ROS) production contribute to muscle weakness and atrophy in mice with the I4895T mutation in RyR1 and that both the weakness and the atrophy are reversed by the treatment of these IT mice with a chemical chaperone, 4-phenylbutyrate (4PBA).

\section{Results}

Generation and phenotyping of IT mice. We generated mice with the I4895T mutation in RyR1 as described in Supplementary Fig. 1. These mice were backcrossed $>10$ generations onto a C57B6/J background. The I4895T mutation was homozygous lethal and, therefore, all experiments were performed with the heterozygous I4895T male mice (designated IT) and wild type (WT) littermates. Parallel studies with female mice are ongoing. When an adequate number of WT littermates were not available we used carefully aged matched WT mice from our colony on a C57B6/J background.

IT mice on a normal chow diet weighed less than their WT littermates with the greatest difference manifesting at young ages (Fig. 1a). However, after 6 weeks on a high fat diet, the IT mice gained more weight (Fig. 1b) than WT mice. IT mice ran significantly less on monitored exercise wheels (Fig. 1c) and performed poorly in wire hang tests compared to WT littermate controls (Fig. 1d). We also assessed muscle-specific (soleus, extensor digitorum longus (EDL) and diaphragm) changes in function. For clarity, we present primarily soleus data in Figs 1-8. Similar results were obtained in EDL and diaphragm muscles and these data are shown in Supplementary Figs 2 and 3, respectively. In younger mice the force-frequency curves were shifted to the right $(\sim 9 \mathrm{~Hz}, 10$-week-old mice, Fig. 1e) without a major change in maximal specific force production. In older IT mice (30 weeks of age) maximal force was decreased (see Fig. 8d). However, fatigue was not significantly altered by the IT mutation (Fig. 1f).

While the soleus from mice with the IT mutation displayed slightly more IIb/x fibres (Supplementary Fig. $4 a-c$ ), we found a significant decrease in the cross-sectional area (CSA) of all fibre types at three different ages (Fig. 1g-j). The frequency distributions of fibres with different CSAs from soleus, EDL and diaphragm muscles of IT and WT mice at different ages are shown in Supplementary Fig. 5.

$\mathrm{Ca}^{2+}$ handling and oxidative stress. Loy et al. ${ }^{4}$ found that the IT mutation reduced $\mathrm{Ca}^{2+}$ permeation through RyR1 without changing SR lumenal $\mathrm{Ca}^{2+}$ levels. We evaluated the effects of the IT mutation on $\mathrm{Ca}^{2+}$ handling in isolated flexor digitorum brevis (FDB) fibres using the low affinity $\mathrm{Ca}^{2+}$ sensor, Mag-Fluo 4. We found a small reduction in the $\mathrm{Ca}^{2+}$ transients at all stimulation frequencies in fibres from IT compared to WT littermate mice (Fig. 2a). The resting cytosolic $\mathrm{Ca}^{2+}$ levels (measured with the high affinity $\mathrm{Ca}^{2+}$ sensor, Fura-2) were lower in FDB fibres from IT compared to WT littermate mice (Fig. 2b), suggesting that this mutation does not lead to $\mathrm{Ca}^{2+}$ leak. However, the relative difference in the amplitudes of the $\mathrm{Ca}^{2+}$ transients in IT and WT fibres increased with repetitive, fatiguing stimulations $(100 \mathrm{~Hz})$, suggesting the possibility of activitydependent modifications of RyR1 activity (Fig. 2c).

ER-mitochondrial-associated membranes (MaMs) are sites of $\mathrm{Ca}^{2+}$, phospholipid and ROS exchange between the ER and mitochondria ${ }^{16,17}$. Similar structures designated SR mitochondrial-associated membranes (SR-MaMs) are found in skeletal muscle ${ }^{18}$ and are thought to play a role in matching contraction-associated energy demands with ATP production via $\mathrm{Ca}^{2+}$ signalling to mitochondria ${ }^{19}$. Decreased resting cytosolic $\mathrm{Ca}^{2+}$ levels and reduced $\mathrm{Ca}^{2+}$ transient amplitudes would be expected to decrease mitochondrial $\mathrm{Ca}^{2+}$ uptake in IT compared to WT fibres. To determine if this is indeed the case, we attempted to assess the effects of the IT mutation on $\mathrm{Ca}^{2+}$ uptake by mitochondria at the SR-MaMs using Rhod2 (refs 20,21). However, any changes in passive $\mathrm{Ca}^{2+}$ uptake into mitochondria in IT and WT fibres were below the detection limits of Rhod2. Since even small increases in mitochondrial $\mathrm{Ca}^{2+}$ uptake are associated with increased mitochondrial ROS production ${ }^{22,23}$, we used the mitochondrial ROS sensor, MitoSOX ${ }^{24}$; RU360, an inhibitor of the mitochondrial $\mathrm{Ca}^{2+}$ uniporter (MCU); and Xestospongin C, an inhibitor of inositol 1,4,5-trisphosphate receptors $^{25}$ (IP3R), to determine if mitochondrial ROS production was different in WT and IT FDB fibres. The interfibrillar mitochondria in the FDB fibres of the IT mice displayed markedly increased ROS production and this increase in ROS in the IT fibres was blocked by RU360 (Fig. 2d,e) and 
a
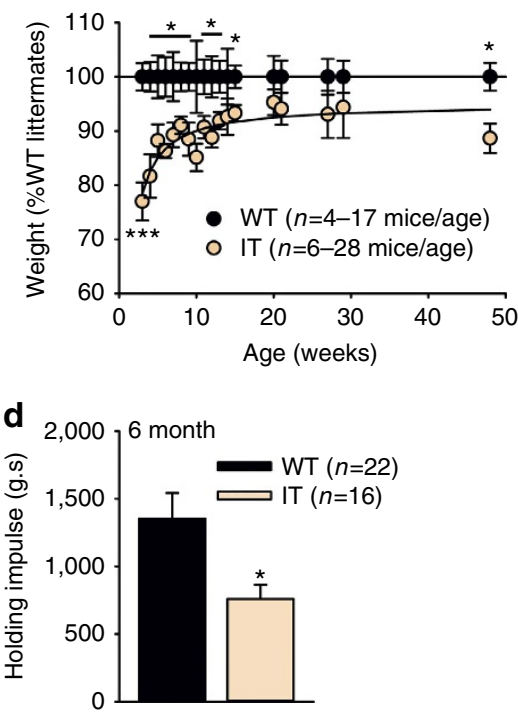

g
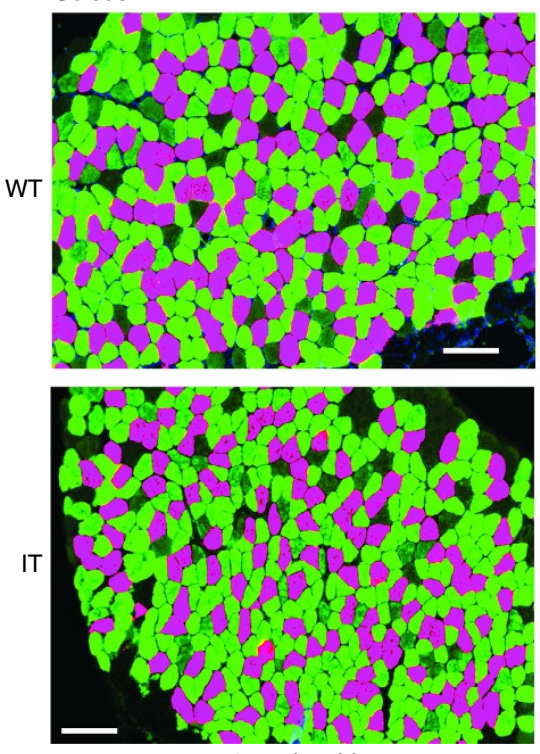

4 weeks old

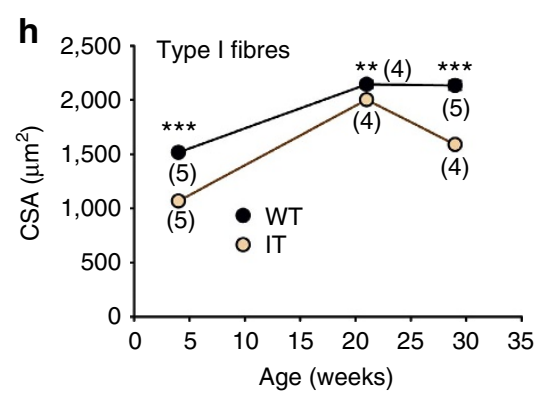

b

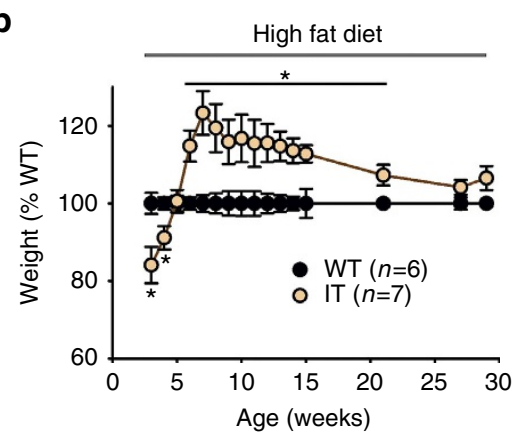

e

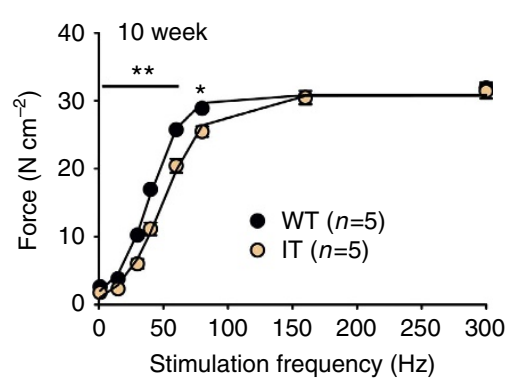

c

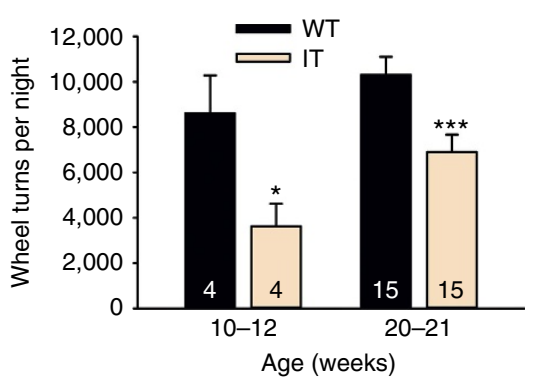

f

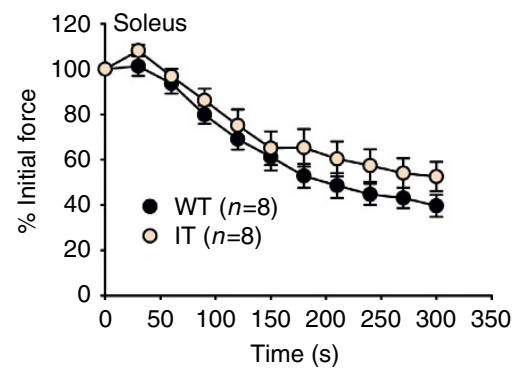

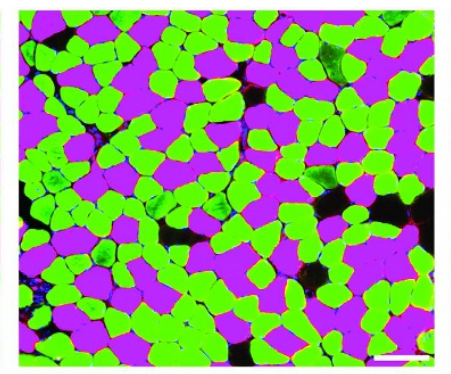
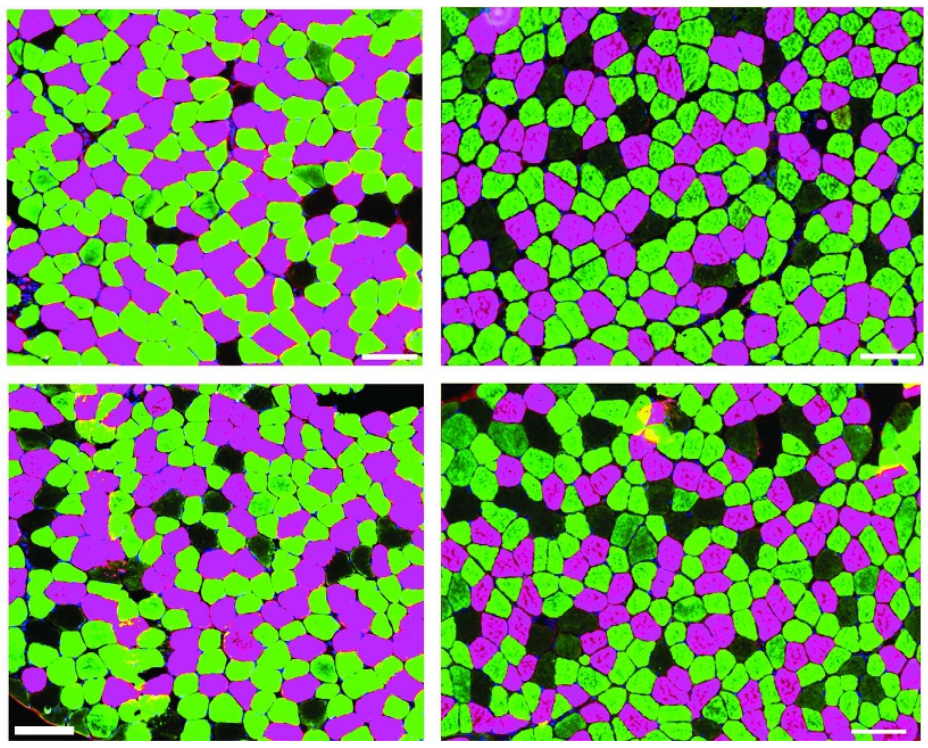

20 weeks old
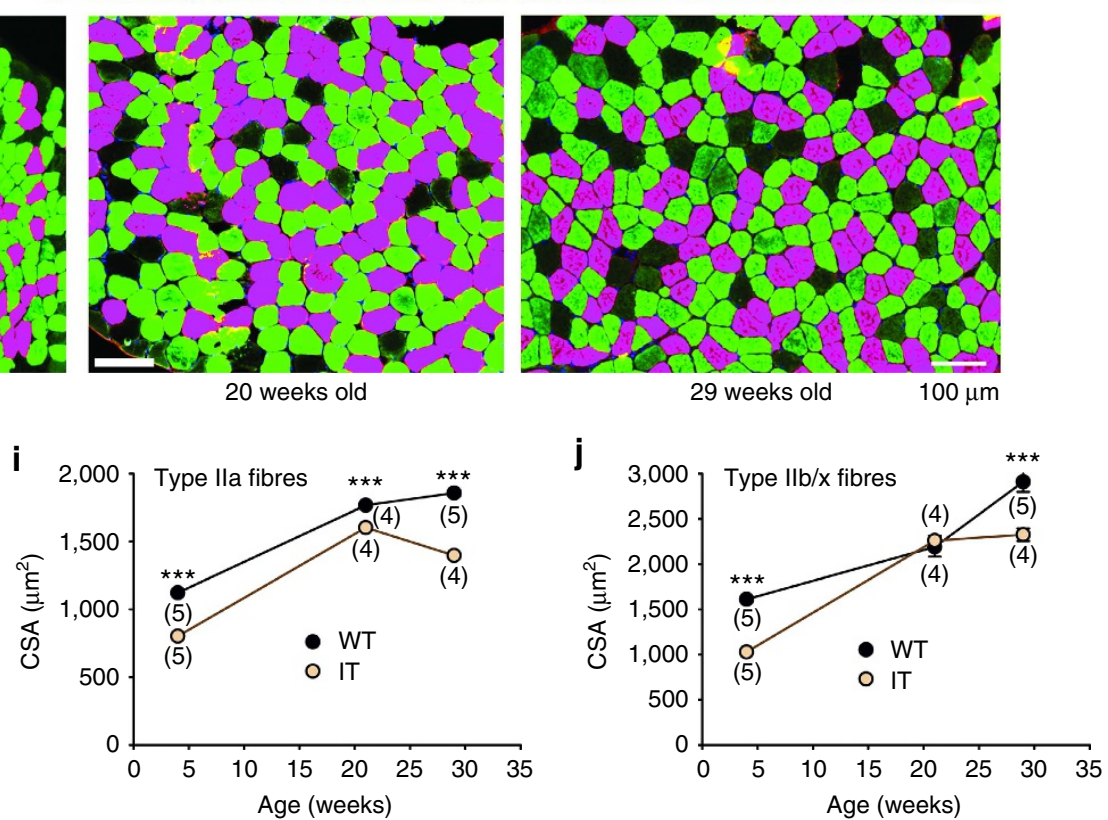

Figure 1 | Phenotype of $\mathbf{1 4 8 9 8 T}$ mice. (a) Body weight of IT and WT littermates on a normal chow diet as a function of age. (b) Body weight of mice on a high fat diet as a function of age. (c) Monitored running in WT and IT mice at two ages. (d) Holding impulse (Body weight $(\mathrm{g}) \times$ the maximal length of time that mice (29 weeks) hang from a wire without falling. (e) Force frequency for the soleus muscle of 10-week-old WT and IT mice. (f) Fatigue in the soleus of 10-week-old WT and IT mice (plotted as \% initial force) with $15 \mathrm{~Hz}$ stimulation train. (g) Representative fibre type immunostaining images in soleus muscles of IT and WT at different ages using specific myosin heavy chain antibodies. Figures shown are pseudo-coloured magenta for type I fibres, green for type Ila and black for type Ilb/x. Scale bars are $100 \mu \mathrm{m}$. (h-j) Analysis of fibre CSA of different fibre types in the soleus of IT and WT. Distributions of CSAs for these muscles are shown in Supplementary Fig. 5. $n$ is the number of mice used in each experiment. Data are shown as mean \pm s.e.m. ${ }^{\star} P<0.05$, ${ }^{\star \star} P<0.01,{ }^{* \star \star} P<0.001$. For statistical analysis we used a two-tailed Student's $t$-test, with differences considered significant at $P<0.05$. 


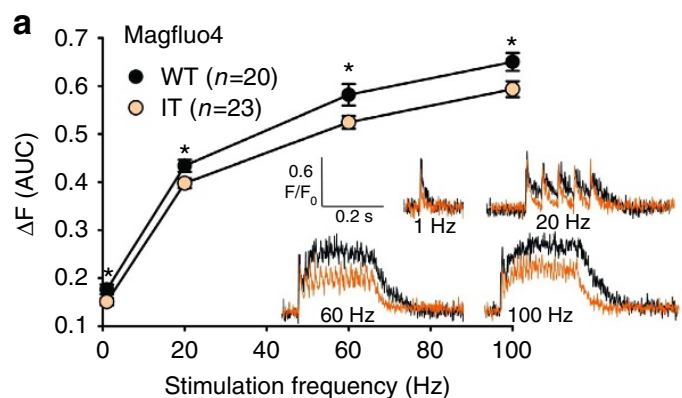

d

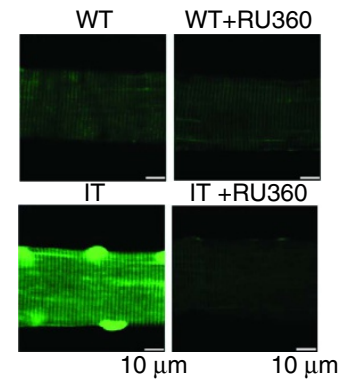

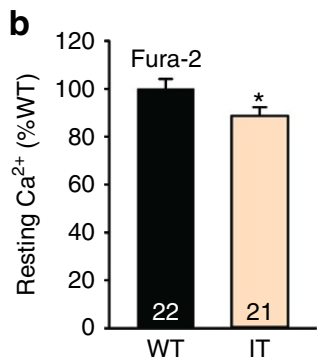

e

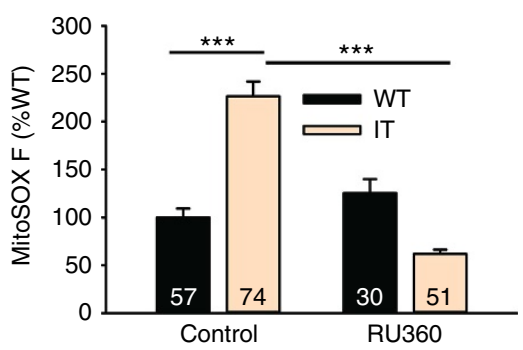

C

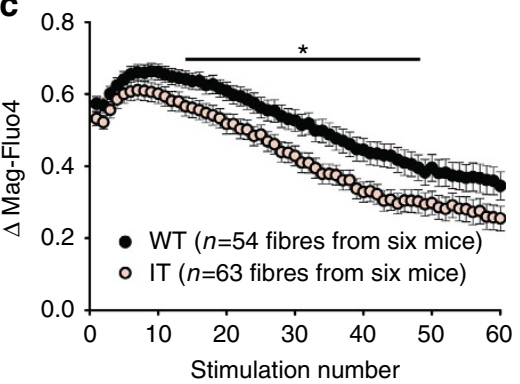

f

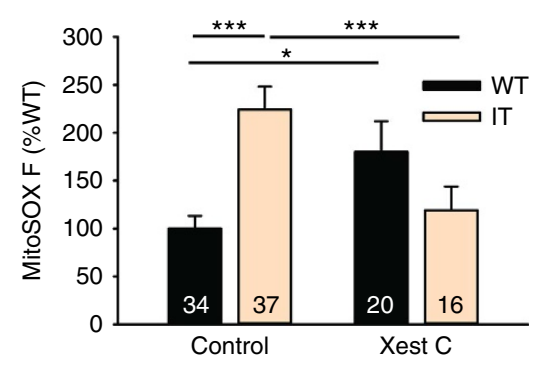

g
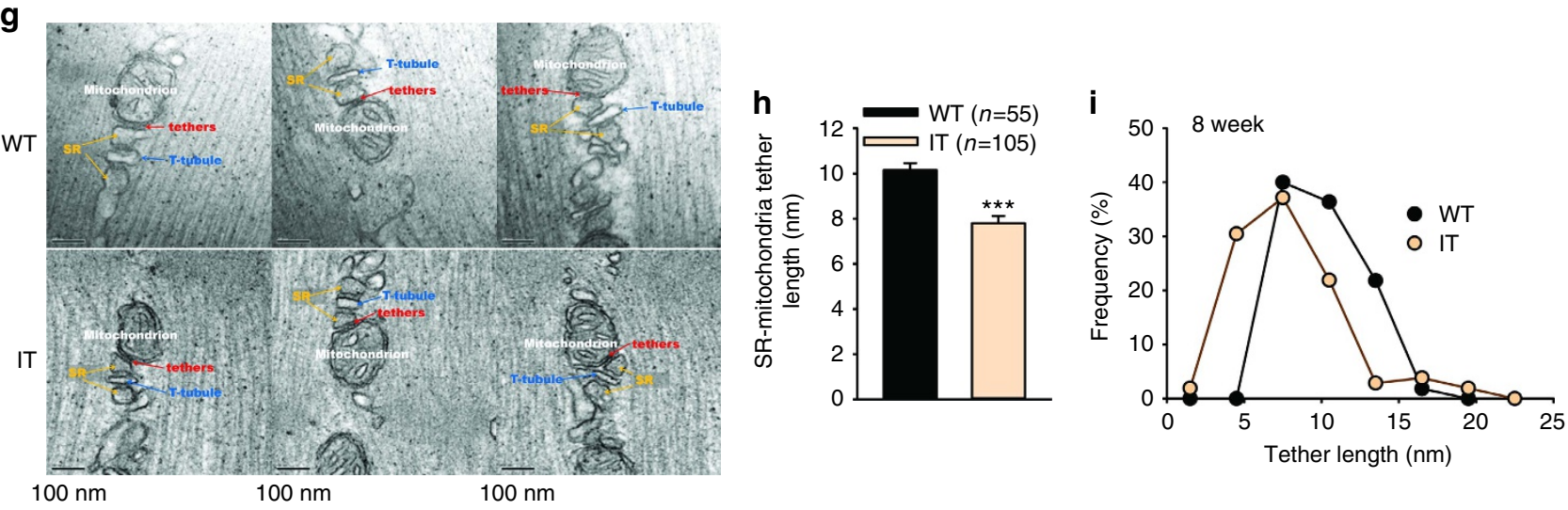

Figure $2 \mid \mathbf{C a}^{2}+$ handling. (a) Analysis of $\mathrm{Ca}^{2}+$ release in FDB fibres (area under the curve, AUC) at different stimulation frequencies using Magfluo4. Inset: Representative $\mathrm{Ca}^{2}+$ transients at different stimulation frequencies. $n$ is the number of fibres from at least three different mice. (b) Resting cytosolic $\mathrm{Ca}^{2}+$ concentration relative to that of WT fibres assessed with Fura-2 in FDB fibres. $n$ is the number of fibres from at least three different mice. (c) Analysis of the amplitudes of the $\mathrm{Ca}^{2+}$ transients in FDB fibres from WT and IT mice with repetitive $100 \mathrm{~Hz}(200 \mathrm{~ms}$ train/1.5 s) stimulations. (d) Representative fibres loaded with MitoSOX from WT and IT mice with and without RU360 treatment. Scale bars are $10 \mu \mathrm{m}$. (e) Analysis of images in panel D. MitoSOX fluorescence in FDB fibres (with and without RU360 to inhibit MCU) from WT and IT mice was plotted as \% WT control (littermate, same day). $n$ is the number of fibres from three to four mice. (f) Effect of Xestospongin C on MitoSox fluorescence. (g) Negatively stained EM pictures of SR-MaMs from IT and WT soleus. Bar is $100 \mathrm{~nm}$. (h) Analysis of the average length of the tethers between the SR and the interfibrillar mitochondria. (i) Distribution of tether length in WT and IT soleus. Data are shown as mean \pm s.e.m. ${ }^{\star} P<0.05,{ }^{\star \star} P<0.01,{ }^{\star \star \star} P<0.001$ (two-tailed Student's $t$-test).

Xestospongin C (Fig. 2f). Since resting cytosolic $\mathrm{Ca}^{2+}$ levels were decreased and mitochondrial ROS production was blocked by both RU360 and Xestospongin C in IT fibres, the increased ROS production is likely to be occurring as the result of increased mitochondrial $\mathrm{Ca}^{2+}$ uptake at ER/SR-MaMs. The lack of an effect of RU360 and Xestospongin C in WT fibres suggests that, under normal conditions in WT fibres, $\mathrm{Ca}^{2+}$ release to mitochondria at the ER/SR-MaMs is minimal. The absence of a major contribution of mitochondrial $\mathrm{Ca}^{2+}$ uptake via the microdomain at the ER/SR MaMs in WT skeletal muscle is consistent with the finding that skeletal muscle interfibrillar mitochondria primarily take up $\mathrm{Ca}^{2+}$ in response to large global myoplasmic $\mathrm{Ca}^{2+}$ changes during repetitive stimulation ${ }^{26-28}$. Our data suggest that persistent ER stress drives $\mathrm{Ca}^{2+}$ uptake and ROS production by mitochondria at the ER/SR-MaMs. IP3Rs are the $\mathrm{Ca}^{2+}$ release channels enriched in ER-MaMs ${ }^{29}$ in other cell types and our finding of blockage of mitochondrial ROS production by Xestospongin C suggests that IP3Rs are also the $\mathrm{Ca}^{2+}$ release channels at the ER/SR-MaMs in skeletal muscle. The IT mutation appears to be increasing $\mathrm{Ca}^{2+}$ uptake, leading to increased ROS production by mitochondria at the ER/SR-MaMs despite decreases in both resting bulk cytosolic $\mathrm{Ca}^{2+}$ and voltage gated $\mathrm{Ca}^{2+}$ release. The IT mutation in RyR1 did not greatly alter the overall structure of the SR-MaMs (Fig. 2g), but the tethers between the SR and mitochondria were significantly shorter at the IT SR-MaMs (Fig. 2h,i). The decreased distance from the SR to the mitochondria could contribute to the increased mitochondrial $\mathrm{Ca}^{2+}$ uptake in IT muscle; however, further analyses of the structure and function of the ER/SR MaMs in IT muscle is needed.

We also assessed the expression levels of $\mathrm{Ca}^{2+}$ handling proteins in the IT mice. We found that the levels of $\mathrm{Ca}^{2+}$ 
a

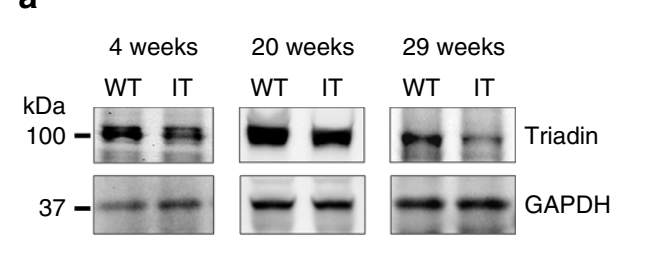

C
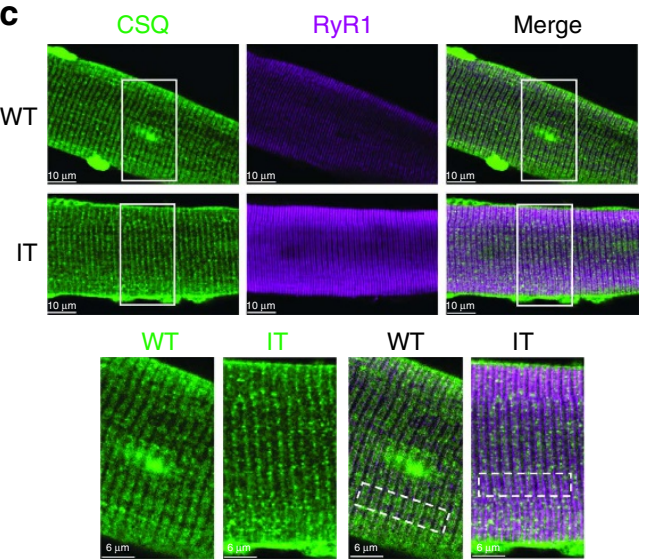

IT

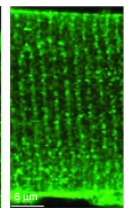

WT
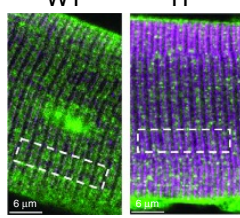

e

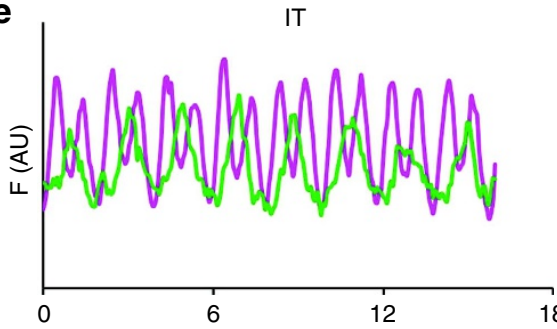

b
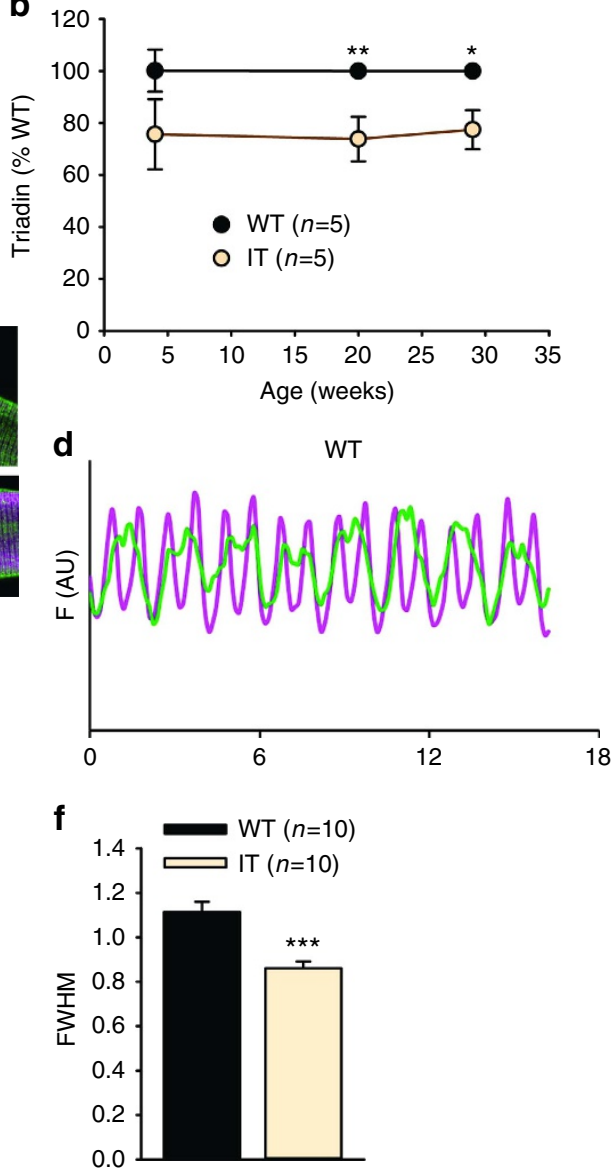

Figure 3 | Effect of the IT mutation on triadin and CSQ. (a) Representative western blots of triadin levels in soleus muscle from WT and IT mice at different ages. (b) Analysis of triadin levels. (c) RyR1 and CSQ localization in FDB fibres by immunocytochemistry. Magenta: RyR1, Green: CSQ. (d,e) Line scans of CSQ and RyR1 immunostaining in FDB fibres of IT (e) and WT (d) mice. (f) Analysis of the fluorescence profile of CSQ in WT and IT fibres, FWHM is full width at half maximum. Data are shown as mean \pm s.e.m. ${ }^{\star} P<0.05,{ }^{\star \star} P<0.01,{ }^{\star \star \star} P<0.001$ (two-tailed Student's $t$-test).

handling proteins (RyR1, $\mathrm{Ca}_{\mathrm{V}} 1.1$, SERCA2, calsequestrin (CSQ)) in the EDL, diaphragm and soleus were, for the most part, not significantly different in muscles of WT and IT mice (Supplementary Figs 2f,g; 3f,g; 4d,e), but, as expected from the small fibre type shifts, there was a small increase in SERCA1 in the diaphragm (Supplementary Fig. 3g). The IT mutation in RyR1 is located in the selectivity filter but is close to the lumenal binding sites for triadin ${ }^{30,31}$. Triadin is required to maintain CSQ at the junctional SR in close proximity to RyR1 (ref. 32) and to optimize excitation-contraction coupling ${ }^{30,33}$. We found a decrease in the amount of triadin in IT muscle (Fig. 3a,b) at three different age groups (4, 20 and 29 weeks old) and an alteration in the localization of CSQ to the junctional SR (Fig. 3c-f). Our data suggest that the IT mutation is altering the interactions of RyR1 with proteins in the SR lumen.

Consequences of persistent $\mathrm{Ca}^{2+}$ uptake into the mitochondria. Persistent elevations in mitochondrial $\mathrm{Ca}^{2+}$ uptake and ROS production at the ER/SR-MaMs would be expected to cause mitochondrial damage. Consistent with increased mitochondrial damage, mitochondrial protein content was decreased (20-weekold mice, Fig. 4a,b; Supplementary Figs 2h,i; 3h,i) and cytosolic cytochrome $\mathrm{c}$ was increased in IT muscle (Fig. 4c,d), suggesting that there has been mitochondrial permeability transition pore opening (mPTP).
Excessive mitochondrial $\mathrm{Ca}^{2+}$ uptake at the ER-MaMs also drives the activation of proapoptotic pathways ${ }^{34-36}$. In support of this, we found: (a) increased apoptotic nuclei (Fig. 4e,f), (b) increased cleaved caspases 3, 9 and 12 (Fig. 4g,h), (c) increased caspase 3 activity (Fig. 4i) and (d) marked elevation of the tumour suppressor and proapoptotic protein, p53, compared to WT littermates (Fig. 4j,k). These findings suggest that the persistent increases in mitochondrial $\mathrm{Ca}^{2+}$ and ROS production cause both mitochondrial damage and elevation of proapoptotic pathways, thereby contributing to the muscle dysfunction.

The IT mutation causes ER stress. The above findings raise the question of what drives increased mitochondrial $\mathrm{Ca}^{2+}$ uptake/ ROS production at the ER/SR-MaMs, leading to damaged mitochondria. The $\mathrm{SR}$ is an extensive, specialized domain of the ER with a continuous intralumenal space ${ }^{37}$. Physiological stressors ${ }^{38}$ such as oxidative stress, glucose deprivation, inflammatory cytokines, free fatty acids, and other factors that regulate ER function, can cause the accumulation of unfolded/ misfolded proteins that trigger an unfolded protein response (UPR) and ER stress. ER stress/UPR activated signalling, in turn, decreases protein synthesis, upregulates molecular chaperones to facilitate protein folding, and strengthens the contact between the ER and mitochondria to alleviate ER stress, increase ATP production and promote survival ${ }^{39}$. However, persistent or 

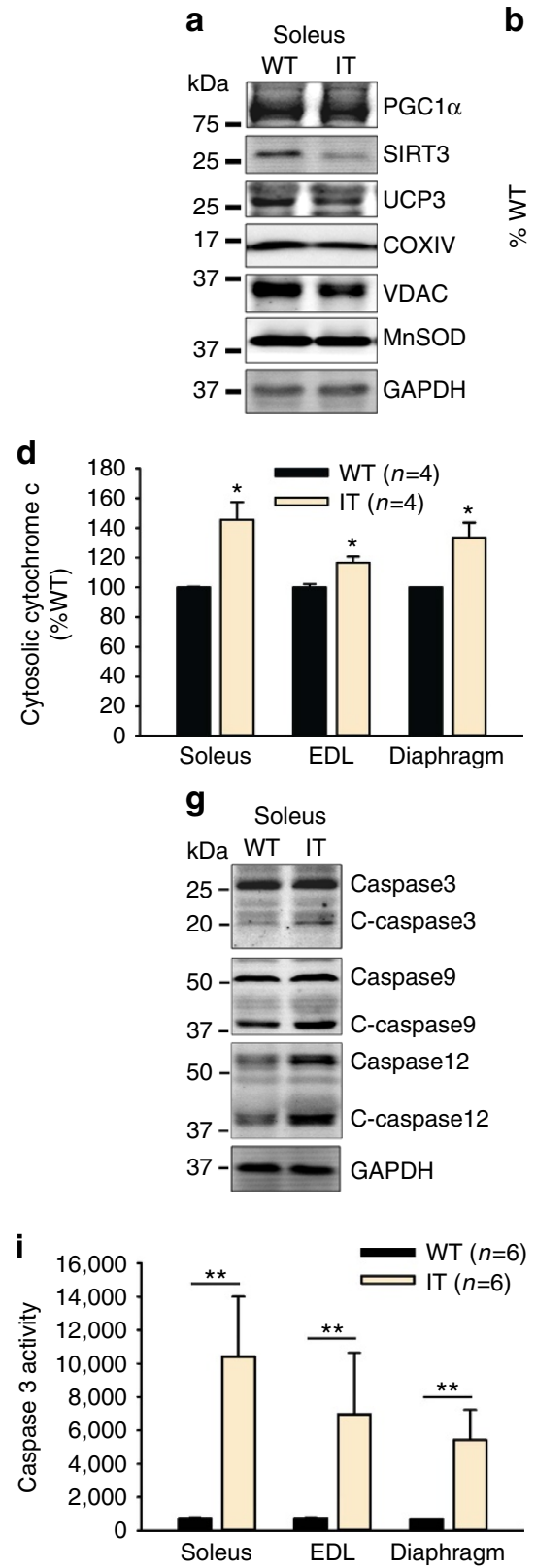

b
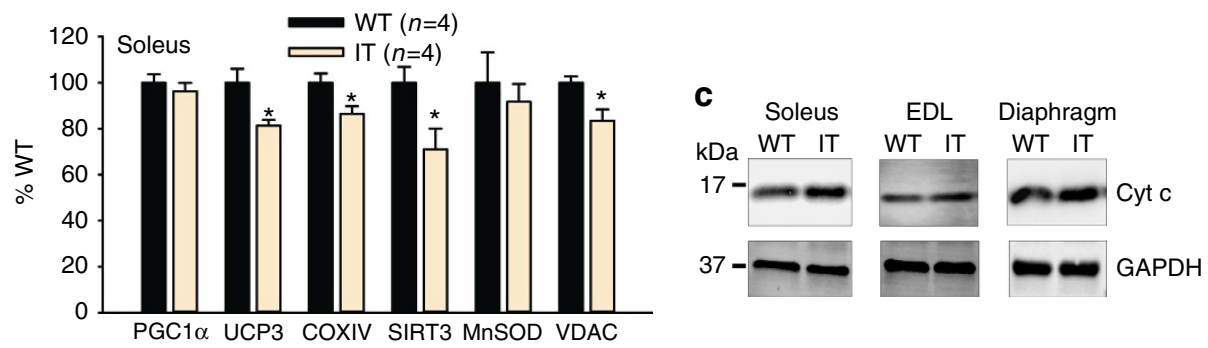

e
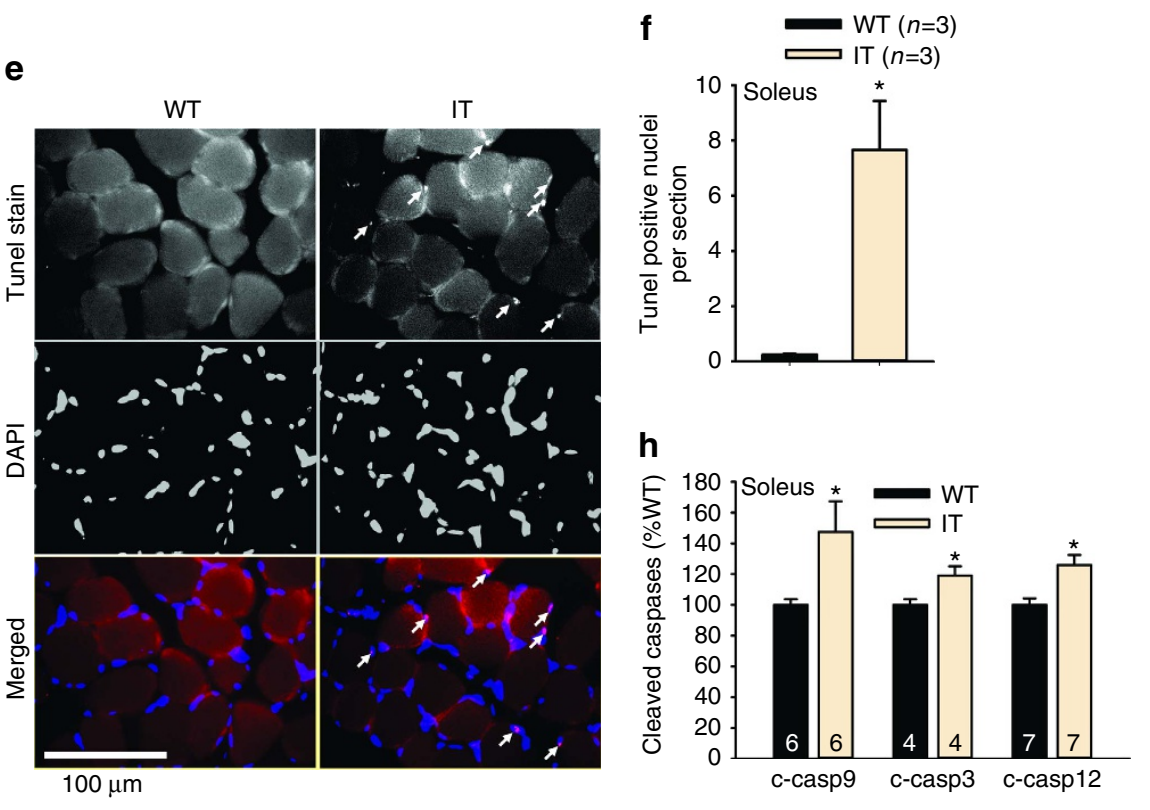

h

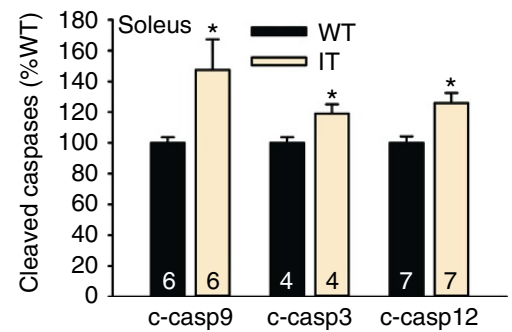

j

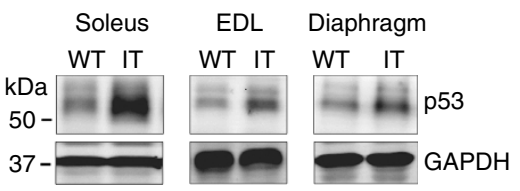

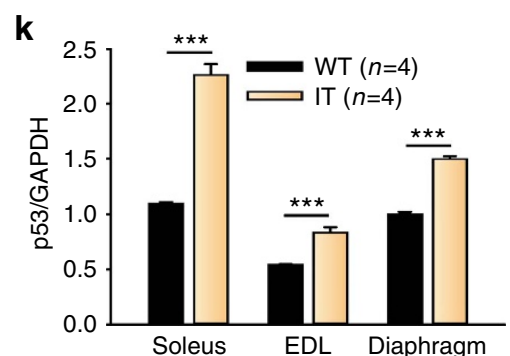

Figure 4 | Mitochondrial damage and activation of proapoptotic pathways in the muscle of IT mice. (a) Representative western blots for mitochondrial proteins in homogenates of soleus muscles from WT and IT mice. (b) Analysis of changes in mitochondrial proteins in homogenates of the soleus muscle of IT compared to WT littermate mice. (c) Representative western blots of cytosolic cytochrome c in soleus from IT and WT mice. (d) Analysis of cytosolic cytochrome c in the soleus of WT and IT mice. (e) TUNEL staining for apoptotic nuclei. Second row of the panel stained for nuclei with DAPI. Third row of the panel is the merged image. (f) Analysis of the number of TUNEL positive nuclei per section. (g) Representative western blots for caspases. (h) Analysis of cleaved caspases. (i) Assay for caspase 3 activity. (j) Representative western blot for p53 in muscles of IT and WT littermates. (k) Analysis of p53 levels in soleus, EDL and diaphragm muscles of WT and IT muscle. $n$ is the number of mice used in each experiment. Data are shown as mean \pm s.e.m. ${ }^{\star} P<0.05$, ${ }^{\star \star} P<0.01,{ }^{\star \star \star} P<0.001$ (two-tailed Student's $t$-test). GAPDH loading controls are below each set of blots from a single gel.

unresolved ER stress further increases mitochondrial $\mathrm{Ca}^{2+}$ uptake $^{34}$ and ROS production, induces MPTP, and drives apoptosis ${ }^{40}$. Both ablation of triadin and a mutation in CSQ that leads to its mislocalization are associated with ER stress in cardiac muscle ${ }^{41,42}$. The decreased triadin, CSQ mislocalization, increased mitochondrial ROS production, and elevation of proapoptotic markers suggested that the muscle of IT might be displaying persistent ER stress/UPR and this was further explored.

We found that proteins associated with ER stress were significantly elevated in muscles (Fig. 5a-d) of IT compared to
WT littermate mice at all ages tested. ER stress-mediated UPR is orchestrated by the action of three signalling proteins, IRE1 $\alpha$ (inositol requiring enzyme 1), PERK (protein kinase RNA (PKR)like ER kinase) and ATF6 (activating transcription factor $6 \alpha)^{43}$. Cleaved ATF6 $\alpha$ (the active form of this transcription factor) and the downstream target of ATF6 $\alpha$, CHOP (transcriptional factor C/EBP homologous protein), were both elevated. eIF2A (eukaryotic translation initiation factor $2 \alpha$ ) is the downstream target of PERK and the ratio of p-eIF2 $\alpha$ to eIF2 $\alpha$ was increased in IT muscle. We also analysed the alternative splicing (regulated by IRE1 $\alpha$ ) of XBP1 (X box-binding protein 1, 
a

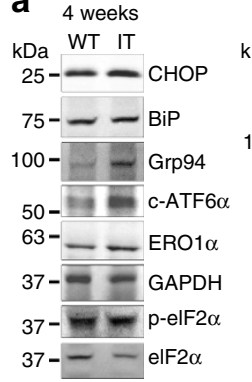

C

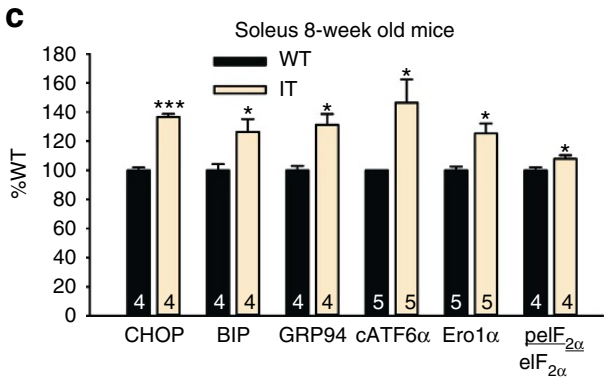

8 weeks

$\mathrm{kDa}$ WT IT

$25-\equiv=$ CHOP

$75--\mathrm{BiP}$

$100--$ Grp94

37- GAPDH

63- - Ero1 $\alpha$

37- - GAPDH

$37-=$ p-elF2 $\alpha$

$37-\ldots-$ p-elF2
$50-7 \rightarrow$ c-ATF6o

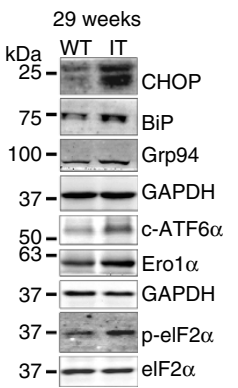

b

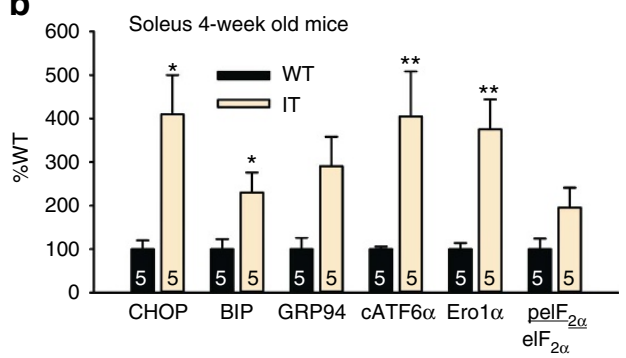

d

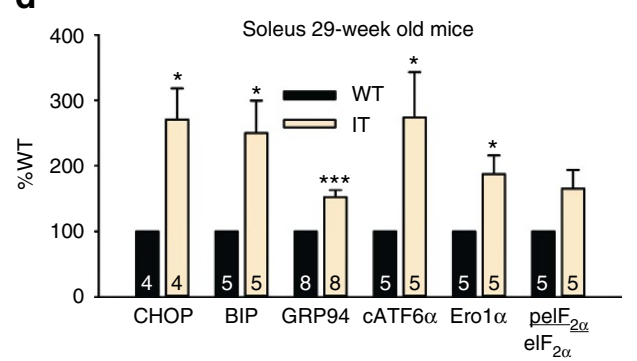

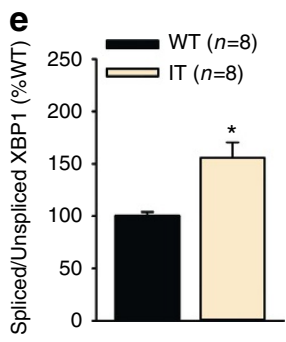
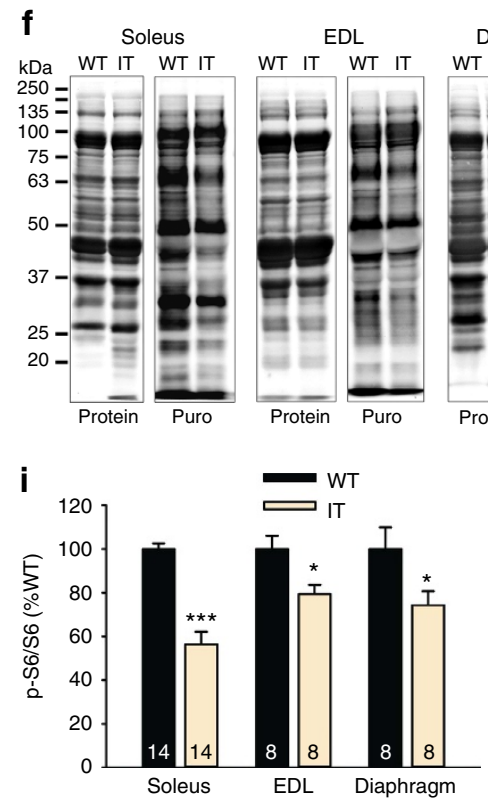

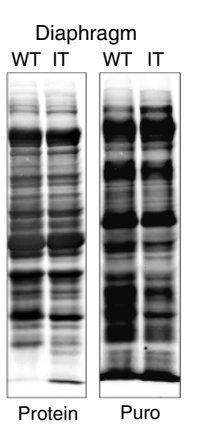

g

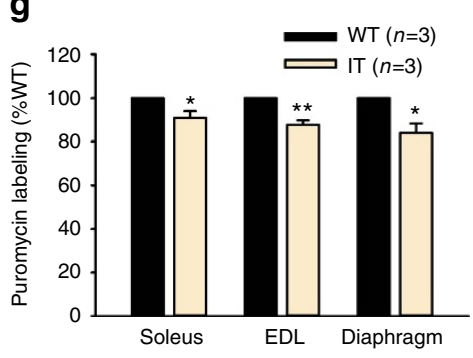

h

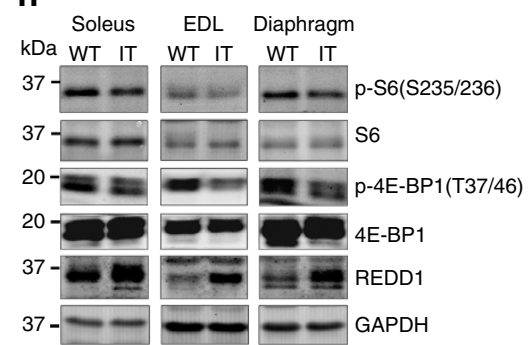

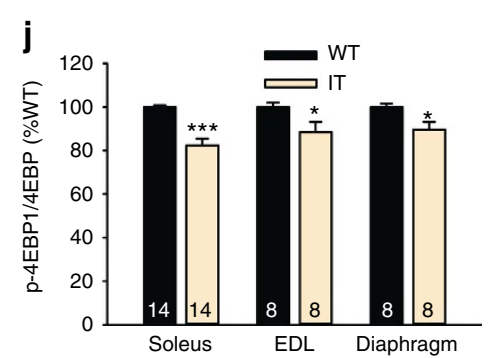

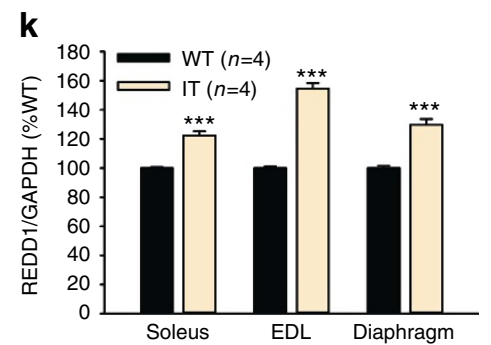

Figure 5 | Evidence of ER stress in IT muscle. (a) Representative western blot of proteins involved in ER stress in the soleus of 4, 8 and 29-week-old IT and WT mice. (b) Analysis of ER stress markers in soleus muscle of 4-week-old IT compared to WT littermate mice. (c) Analysis of ER stress markers in soleus muscle of 8-week-old IT compared to WT littermate mice. (d) Analysis of ER stress markers in soleus muscle of 29-week-old IT compared to WT littermate mice. (e) Analysis of XBP1 splicing by qRT-PCR. (f) Representative western blots for newly synthesized puromycin labelled proteins using anti-puromycin antibody for SUnSET analysis. (g) Analysis of protein synthesis by SUnSET. (h) Representative western blot of proteins downstream of mTORC1 activation and REDD1. (i-k) Analysis of changes in proteins downstream of mTORC1 activation and REDD1 in soleus, EDL and diaphragm muscle of IT compared to WT mice. $n$ is the number of mice used in each experiment. Data are shown as mean \pm s.e.m. ${ }^{\star} P<0.05$, ${ }^{\star \star} P<0.01$, ${ }^{\star \star \star} P<0.001$ (two-tailed Student's $t$-test).

a transcriptional activator for many of the UPR target genes), and found an increase in XBP1 splicing in the soleus muscle (Fig. 5e). These findings suggest that all three arms of the ER stress/UPR response are persistently elevated in the muscle of the IT mice.

Persistent ER stress decreases protein synthesis, causes mitochondrial damage and activates proapoptotic pathways ${ }^{44}$. As discussed above, both mitochondrial damage and activation of proapoptotic pathways are occurring in IT muscle (Fig. 4). Protein synthesis, assessed with the SUnSET puromycin technique ${ }^{45,46}$, was decreased in the muscles of IT compared to
WT mice (Fig. 5f,g). Both p-S6/S6 (ribosomal protein S6) and $\mathrm{p}-4 \mathrm{EBP} / 4 \mathrm{EBP} 1$ (eukaroyotic translation iniitation factor 4E-binding protein), markers of mTORC1 activation, were decreased while REDD1 (a negative regulator of mTORC1 activity) was increased in IT muscle (Fig. 5h-k). Decreased protein synthesis is, therefore, likely to contribute to decreased muscle fibre size in the IT mice.

Mitigation of ER stress with a chemical chaperone. If ER stress is a major contributor to the myopathy associated with the IT 

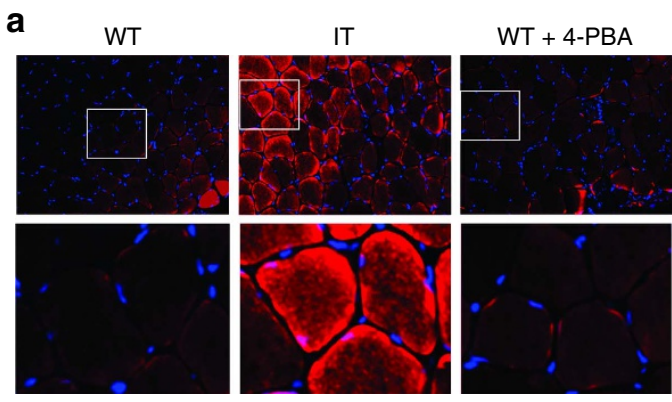

$$
\text { d }
$$
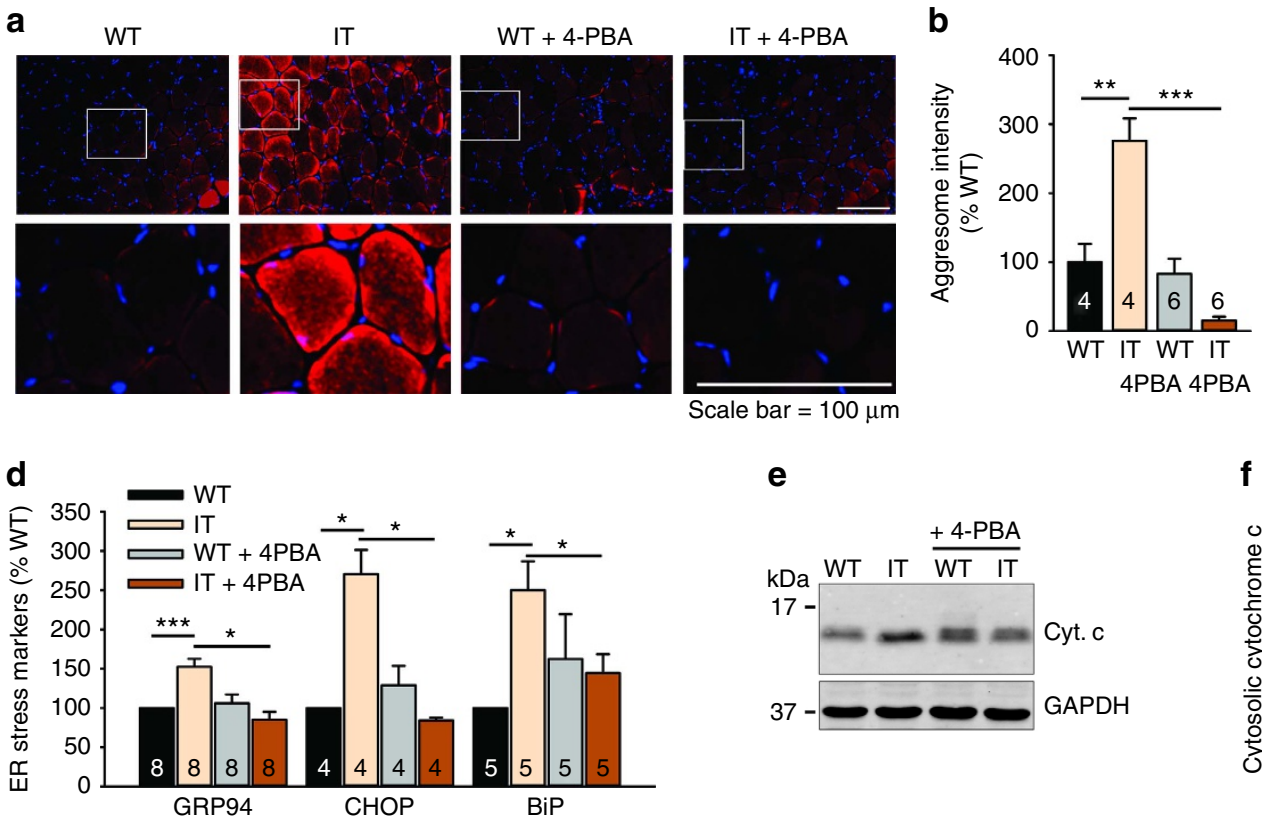

C

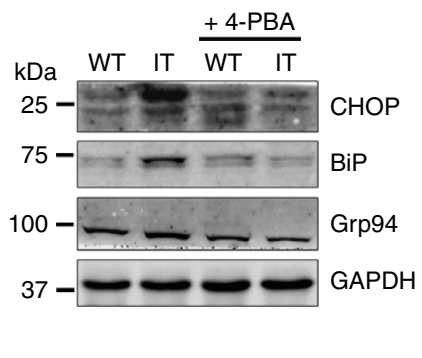

e
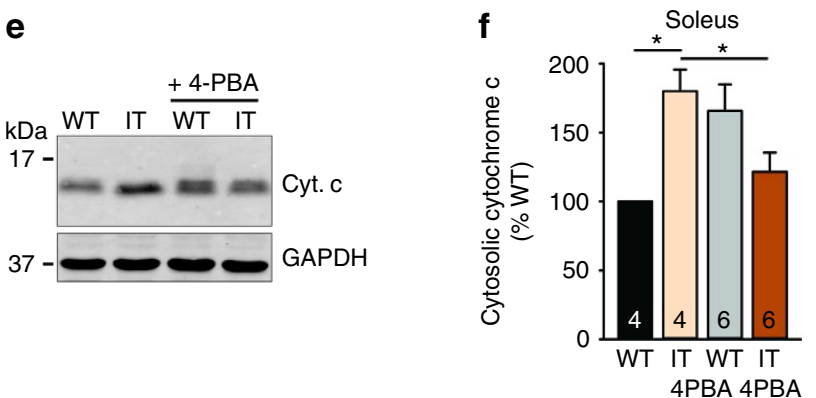

g

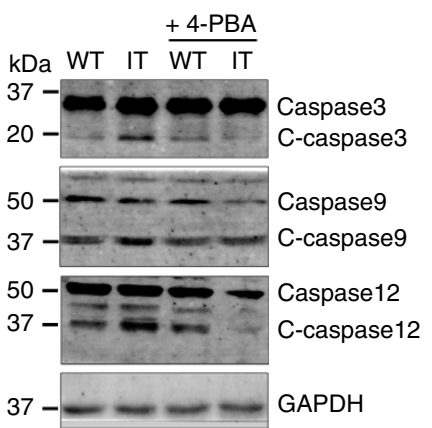

h

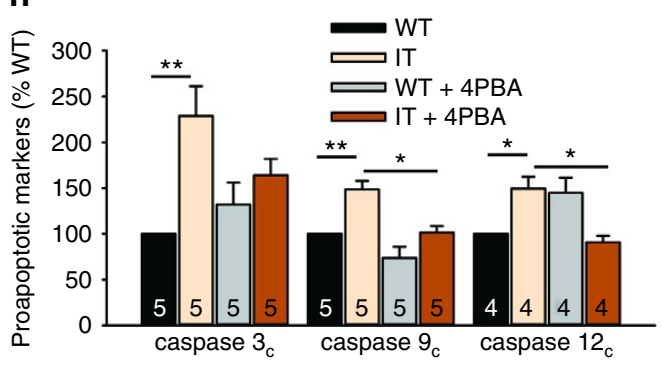

i

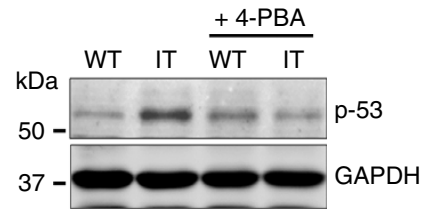

j

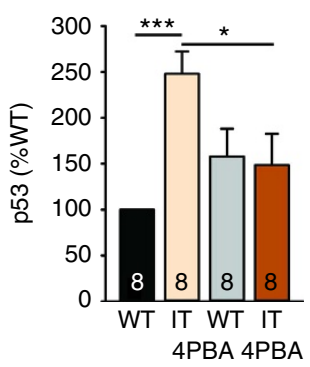

k

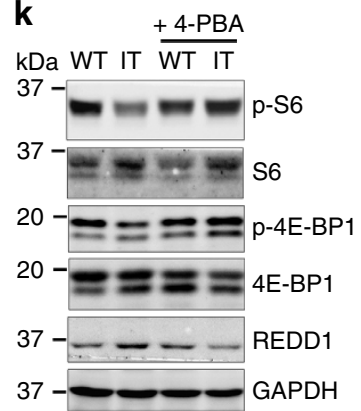

I

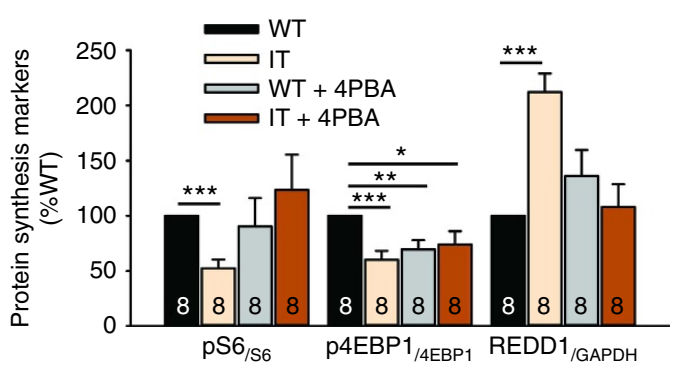

Figure 6 | 4PBA reverses the signalling changes in IT muscle. (a) ProteoStat aggresome staining in the soleus of IT and WT littermates treated for 2 weeks with or without 4PBA. Lower panels are enlarged images boxed in upper panels. Scale bar is $100 \mu$ m. (b) Analysis of aggresome intensity in the soleus with or without treatment of 4PBA for 2 weeks. (c) Representative western blots on ER stress markers from the soleus of WT and IT mice treated for 2 weeks with 4PBA. (d) Effects of 4PBA on ER stress markers. (e) Representative western blot for cytosolic cytochrome c in the cytosolic fraction of soleus from WT and IT mice treated with and without 4PBA for 2-3 weeks. (f) Analysis of cytosolic cytochrome c in muscle from IT and WT littermate mice treated with and without 4PBA. (g) Representative western blot showing the effect of 2-week treatment of 4PBA on cleaved caspases in the soleus. (h) Effects of 4PBA on cleaved caspases in the soleus. (i) Representative western blots of p53 in the soleus of IT and WT littermates without or with 4PBA treatment for 2-3 weeks. (j) The effects of 4PBA on p53 levels in soleus. ( $\mathbf{k}$ ) Representative western blot of proteins downstream of $\mathrm{mTORC1}$ in the soleus of IT and WT littermates \pm 4PBA treatment for 2-3 weeks. (I) The effects of 4PBA on proteins downstream of mTORC1 from $>3$ pairs of IT and WT littermates. Data are shown as mean \pm s.e.m. ${ }^{\star} P<0.05,{ }^{\star \star} P<0.01,{ }^{\star \star \star} P<0.001$ (two-tailed Student's $t$-test).

mutation, then interventions that alleviate ER stress should normalize the altered pathways and improve muscle function. Chemical chaperones aid in protein folding and reduce protein aggregation and oxidative stress (reviewed in ref. 47). Since ER stress, mitochondrial damage and apoptosis are defining features of IT muscle pathology, we sought to determine if restoration of protein folding with a chemical chaperone, $4 \mathrm{PBA}$, would reverse muscle pathology through reduction of ER stress/UPR and oxidative stress. The data shown in Figs 6 and 7 were obtained with mice treated for $>2$ weeks with 4 PBA in the drinking water. 

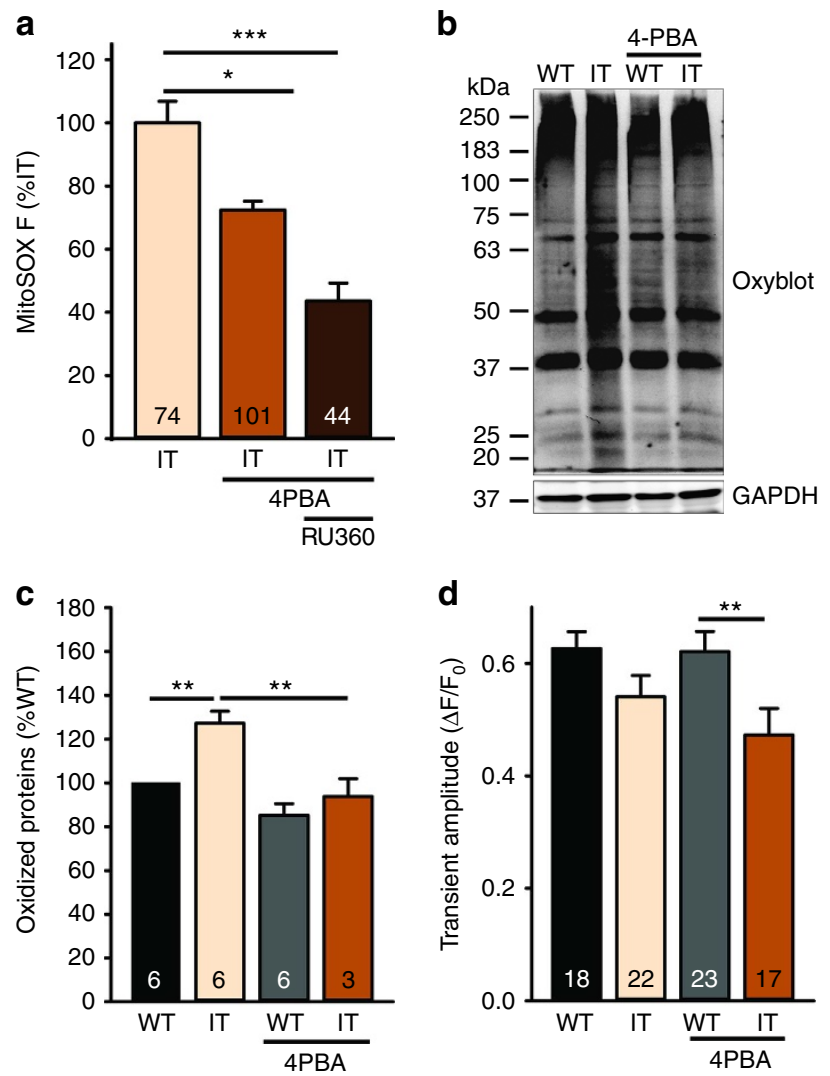

Figure 7 | Effect of 4PBA on mitochondrial ROS and RyR1-mediated $\mathbf{C a}^{2+}$ release. (a) Analysis of MitoSOX fluorescence in IT treated with and without 4PBA for 3 weeks. Plotted as \% IT untreated. Also, shown is the effect of RU360; the number of fibres is in the bar. (b) Oxyblot of soleus homogenates from mice treated with or without 4PBA. (c) Analysis of oxidized proteins in soleus of mice treated with and without 4PBA.

(d) Analysis of the effects of $4 \mathrm{PBA}$ on the amplitude of the $\mathrm{Ca}^{2+}$ transient assessed with Magfluo4. In all panels except $A, n$ is the number of mice used in each experiment. Data are shown as mean \pm s.e.m. ${ }^{\star} P<0.05$, ${ }^{\star \star} P<0.01$, ${ }^{\star \star \star} P<0.001$ (two-tailed Student's $t$-test).

We found that protein aggregates (stained with ProteoStat) (Fig. 6a,b), ER stress markers (Fig. 6c,d), cytosolic cytochrome c (Fig. 6e,f), cleaved caspases (Fig. 6g,h), p53 (Fig. 6i,j), all elevated in IT muscle, were markedly reduced by treating the IT mice with 4PBA. Markers of mTORC1 activation, reduced by the IT mutation, were restored to WT levels by 4 PBA (Fig. 6k,l). Treatment with $4 \mathrm{PBA}$ also reduced muscle protein ubiquitination (Supplementary Fig. 6a,b) and autophagy markers (Supplementary Fig. 6c,d). Collectively, these findings suggest that alleviating ER stress/UPR normalizes the pathways that are altered by the IT mutation.

We next addressed the critical question of whether 4PBA affects ROS production by the interfibrillar mitochondria or restores $\mathrm{Ca}^{2+}$ release via RyR1 (perhaps by facilitating its refolding). Treatment of mice with $4 \mathrm{PBA}$ reduced both ROS production in FDB fibres (Fig. 7a) and oxidized proteins in muscle homogenates of IT mice (Fig. 7b,c). However, 4PBA did not 'fix' the IT associated decrease in SR $\mathrm{Ca}^{2+}$ release via RyR1 (Fig. $7 \mathrm{~d}$ ), suggesting that the $4 \mathrm{PBA}$ is not just functioning to refold RyR1 with the IT mutation.

4PBA improves muscle function in mice with the IT mutation. Clinically, the most important question is whether the treatment of the IT mice with 4PBA improves muscle function and increases muscle fibre size. In 10-week-old mice 4PBA significantly improved wheel running compared to WT mice (Fig. 8a). In 30-week-old mice, 4PBA treatment dramatically improved wire-hang performance in the IT mice (Fig. 8b,c), fully restoring IT function to WT levels. Maximal force generation in the soleus was decreased in muscle isolated from the 30-week-old IT mice compared to their WT littermate controls, but after 4PBA treatment, the muscle force of the IT mice significantly improved and force generation was similar to the muscle of WT mice (Fig. 8d). Serial muscle cross-sections (stained with H\&E, NADH tetrazolium reductase and MHC antibodies) from the 29-week-old IT mice, their WT littermates, and both IT and WT mice treated with 4PBA for 2-3 weeks are shown in Supplementary Fig. 7. If ER stress-driven changes in protein synthesis and protein turnover underlie the decreased fibre size in the IT mice, then chronic treatment with $4 \mathrm{PBA}$ should restore fibre size of IT mice to a WT level. 4PBA produced an impressive increase in fibre size in all muscles tested (Fig. 8e; Supplementary Figs 2n; 3n). The effect of 4PBA on the CSA distributions in the soleus (29-week-old mice) of IT mice is shown in Supplementary Fig. 8.

We also tested the effect of $4 \mathrm{PBA}$ on wire hang performance in old (50 week) IT mice and again found significant improvement (Fig. 8f). 4PBA also increased fibre size in 50-week-old mice (Fig. 8g). We did not detect major ultrastructural defects in IT muscle either with or without 4PBA (Fig. 8h), but 4PBA reduced ER stress markers even in these older mice (Fig. 8i,j) and increased mitochondrial protein content (Fig. 8k,l).

\section{Discussion}

Defining the mechanisms underlying the pathology of RyR1 myopathies is a critical first step towards developing interventions. We explored the mechanisms by which the I4895T mutation, associated with a severe myopathy in humans (I4898T), alters muscle function in mice. In marked contrast to our previous findings in mice with a RyR1 $\mathrm{Ca}^{2+}$ leaky mutation (Y522S in humans, Y524S in mice $)^{5}$, the IT mutation decreases voltage-gated $\mathrm{Ca}^{2+}$ release and resting cytosolic $\mathrm{Ca}^{2+}$ levels but increases ER stress/UPR, enhances $\mathrm{Ca}^{2+}$ uptake/ROS production by interfibrillar mitochondria, activates proapoptotic pathways and decreases protein synthesis. Since persistent ER stress/UPR decreases protein synthesis, strengthens the contact between the ER and mitochondria, increases mitochondrial $\mathrm{Ca}^{2+}$ uptake and ROS production ${ }^{34}$, induces MPTP and drives apoptosis ${ }^{40}$, our findings suggested the possibility that the IT myopathy arises from a persistent elevation in ER stress/UPR. Indeed, we found ER stress/UPR markers were persistently elevated in the muscle of IT mice at different ages.

A critical question is how the IT mutation in RyR1 drives ER stress/UPR. The IT mutation in RyR1 is in close proximity to the amino acids (D4878, D4907 and E4908) involved in the binding of triadin to RyR1 (ref. 30). We found a decrease in triadin and a mislocalization of CSQ in muscle from the IT mice. CSQ polymerization and its interactions with triadin and junctin play a critical role in its localization to the junctional SR where it is in close contact with RyR1 (refs 30-32). Both triadin deficiency and CSQ mislocalization are associated with ER stress in cardiac muscle ${ }^{41,42}$. Hence, we propose that alterations in lumenal SR proteins (triadin and CSQ) occurring as a result of alterations in their interactions with IT mutated RyR1 drive ER stress/UPR in skeletal muscle.

Another consequence of the IT mutation was increased ROS production, which was blocked by Xestospongin C and RU360 (blockers of IP3R and the MCU, respectively), indicating that 
a

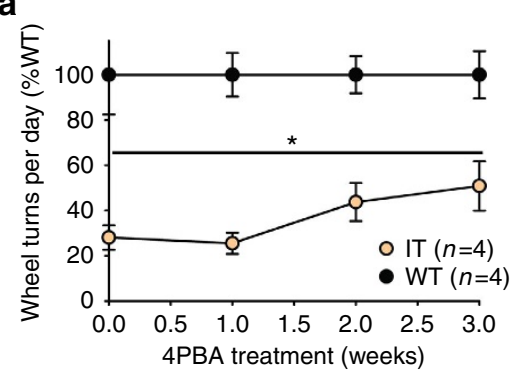

d

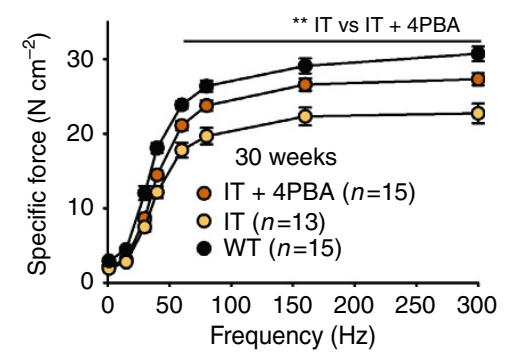

g

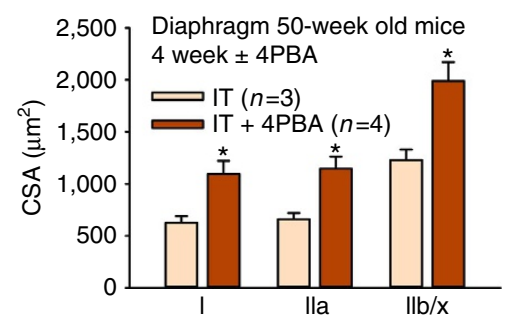

b

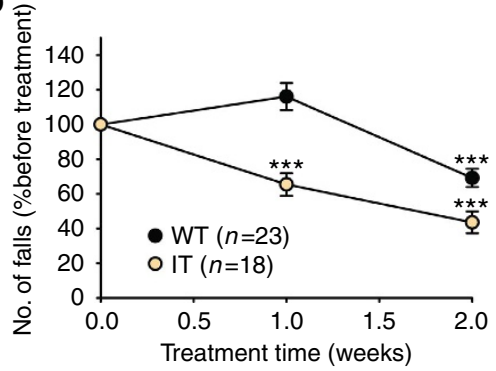

e

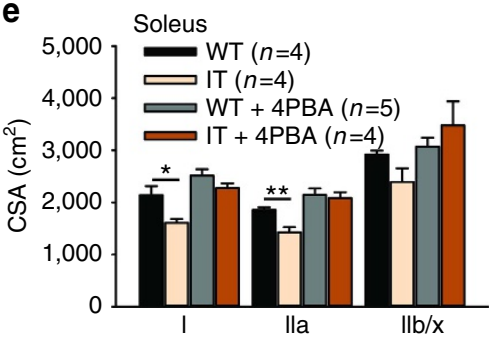

h

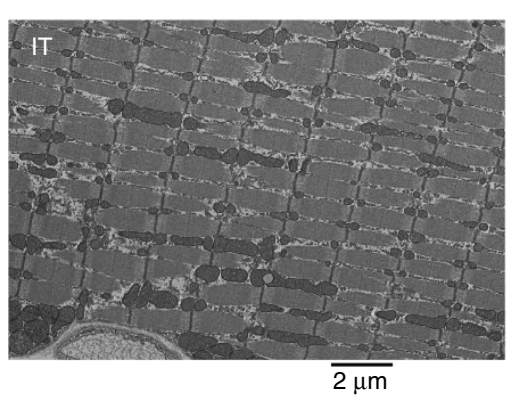

c

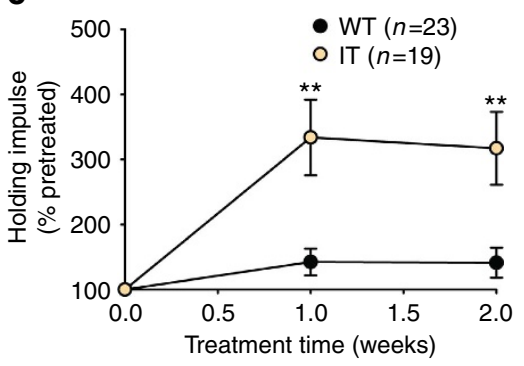

f
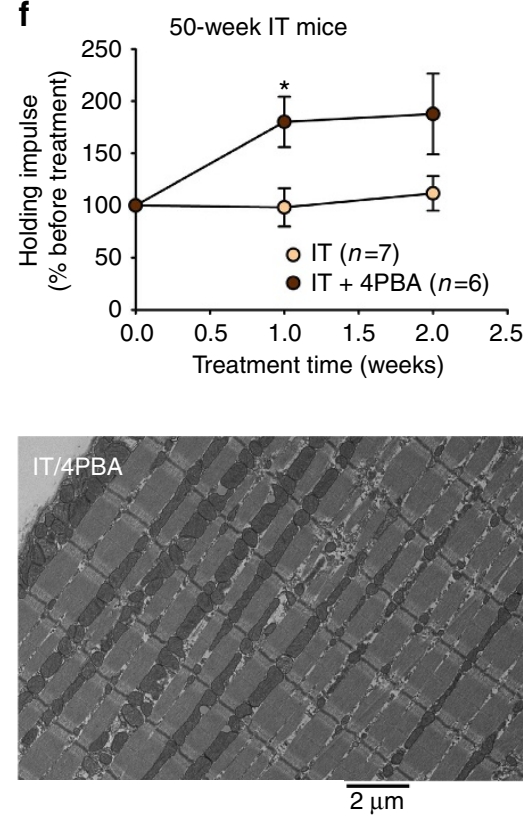

i

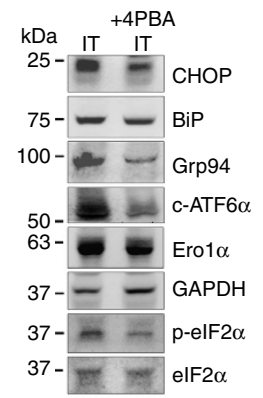

j 120
100
58
$\stackrel{8}{\circ} 60$
240
20

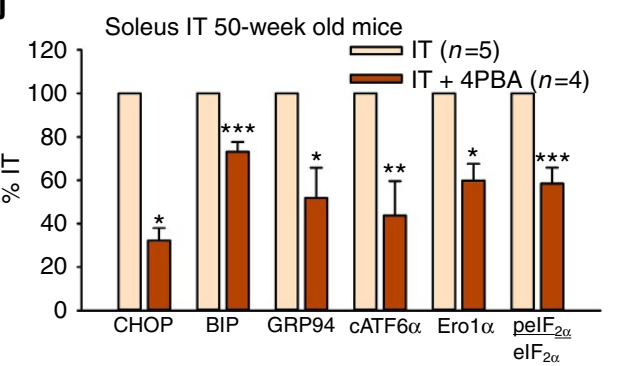

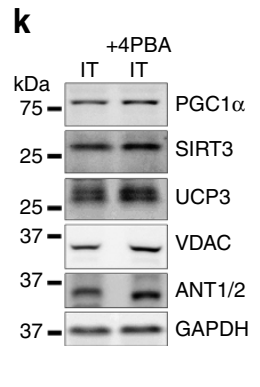

I

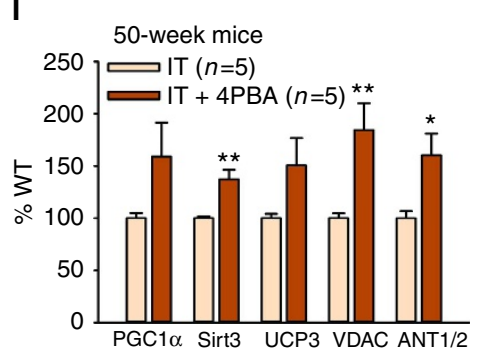

Figure 8 | 4PBA improves muscle CSA and function in IT mice. (a) Wheel turns (\%WT) in 10-12-week-old IT and WT mice treated with 4 PBA. (b) Number of falls (\% before treatment) in a wire hang test (29-week-old mice). Performance is normalized to performance before receiving 4PBA. Significance is assessed by comparing performance after 4PBA with performance before 4PBA. (c) Holding impulse (\%before treatment, holding time $\times$ body weight) for 29-week-old WT and IT mice. (d) Force frequency for WT, IT and IT mice treated with 4PBA for 2-3 weeks (age 25-35 weeks). (e) CSA of the soleus muscle fibres from 29-week-old mice WT and IT mice \pm chronic 4PBA. (f) Holding impulse of 50-week-old IT and WT mice \pm 4 PBA (\% pretreatment). (g) Diaphragm fibre CSA in 50-week-old IT mice treated \pm 4PBA for 4-6 weeks. (h) Negatively stained transmission electron microscopy images of soleus muscle sections from 50 -week-old IT mice treated \pm 4 PBA. Scale bars are $2 \mu \mathrm{m}$. (i) Representative western blot of ER stress proteins in 50 -week-old IT mice treated \pm 4PBA. (j) Analysis of the effects of 4PBA on ER stress markers (plotted as \% untreated IT). (k) Western blot of mitochondrial proteins from 50-week-old IT mice \pm 4PBA treatment. (I) Relative changes in mitochondrial protein content in muscle of 50-week-old IT mice \pm 4PBA treatment. $n$ is the number of mice used in each experiment. Data are shown as mean \pm s.e.m. ${ }^{\star} P<0.05,{ }^{\star \star} P<0.01,{ }^{\star \star \star} P<0.001$ (two-tailed Student's $t$-test) For the data in panels $A, B, C$ and $F$, performance was compared to performance before 4 PBA and used a paired $t$-test.

increased mitochondrial $\mathrm{Ca}^{2+}$ uptake is driving increased ROS production. Since this is occurring under conditions with reduced cytoplasmic $\mathrm{Ca}^{2+}$ levels, the increased mitochondrial $\mathrm{Ca}^{2+}$ uptake is likely to be occurring at the highly specialized microdomains of the ER/SR-MaMs. Consistent with this possibility unresolved ER stress has been shown to increase mitochondrial $\mathrm{Ca}^{2+}$ uptake ${ }^{34}$. In addition, Csordas et al. ${ }^{48}$ found that the spacing between the ER and mitochondria modulates mitochondrial $\mathrm{Ca}^{2+}$ uptake. Shortened distances between the ER and mitochondrial membranes are associated with increased $\mathrm{Ca}^{2+}$ uptake by mitochondria at ER-MaMs and $\mathrm{mPTP}^{49,50}$. The observed decrease in the length of the tethers (tightening of tethers) between the SR and interfibrillar mitochondria in IT fibres could contribute to the increased mitochondrial $\mathrm{Ca}^{2+}$ 
uptake/ROS production, but additional studies of the structure/number of ER-SR-MaMs and direct measurement of mitochondrial $\mathrm{Ca}^{2+}$ uptake are required. Alterations in mitochondrial function could contribute to the metabolic changes detected in the IT mice (increased weight gain on a high fat diet).

Muscle atrophy is a serious problem in most RyR1 myopathies ${ }^{15,51,52}$. The CSA of Type I and IIa fibres in the IT mice are decreased at least in part due to decreased muscle protein synthesis. Reduced protein synthesis is likely to result from ER stress-driven reductions in mTORC1 activity ${ }^{44}$. Increased REDD1 (secondary to elevated p53) ${ }^{53}$, decreased phosphorylation of Akt at S473 by mTORC2, and reduced translational initiation due to the increased phosphorylated eIF2 are likely to contribute to decreased mTORC1 activity.

The finding of persistent ER stress/UPR in the muscle of the IT mice led us to test the effects of a chemical chaperone, $4 \mathrm{PBA}$, for ability to improve muscle function in the IT mice. 4PBA reduces markers of ER stress/UPR, but also increases protein synthesis and muscle mitochondrial protein content and reduces oxidative stress and proapoptotic markers, suggesting that mitochondrial damage and elevation of proapoptotic pathways are secondary to the ER stress/UPR. 4PBA, however, did not restore the amplitude of SR $\mathrm{Ca}^{2+}$ release via RyR1, suggesting that reduced $\mathrm{Ca}^{2+}$ release is not the primary driver of the myopathy. $4 \mathrm{PBA}$ is an FDA-approved drug that facilitates protein folding, in turn, suppressing ER stress-mediated apoptosis by inhibiting eukaryotic initiation factor $2 \alpha$ (eIF2 $\alpha$ ) phosphorylation, CCAAT (highly conserved promoter region of the Grp genes)/enhancerbinding protein homologous protein (CHOP) induction, and caspase-12 activation ${ }^{54}$. $4 \mathrm{PBA}$ also increases gene expression of antioxidants to decrease oxidative stress and protein aggregation in Parkinson disease cell model ${ }^{55}$. In our study, 4PBA dramatically improves muscle function and increases muscle fibre size in the IT mice at all ages tested, strongly supporting our hypothesis that this RyR1 myopathy is an ER stress/UPR disorder.

In summary, our data demonstrate that persistent ER stress/UPR, decreased protein synthesis, mitochondrial ROS production/damage and elevation of proapoptotic markers are defining features of RyR1 myopathy associated with the I4895T mutation in mice, making this myopathy distinct from that of the RyR1 myopathies that arise from $\mathrm{Ca}^{2+}$ leak (model in Fig. 9). These findings emphasize the need to tailor therapeutic approaches for RyR1 myopathies to the mutation and emphasize the importance of distinguishing between RyR1 mutations that cause $\mathrm{Ca}^{2+}$ leak from those that cause persistent ER stress/UPR. It will also be important to determine if ER stress/UPR contributes in any way to the muscle weakness associated with $\mathrm{Ca}^{2+}$ leaky mutations in RyR1. Drugs such as rycals show promise for the $\mathrm{Ca}^{2+}$ leaky mutations ${ }^{6}$, while chemical chaperones and ER stress inhibitors may be better suited for mutations in RyR1 that produce ER stress/UPR. The sequence around amino acid I4898 in RyR1 is a hot spot for mutations that produce myopathies ${ }^{9}$ and these myopathies should all be evaluated for ER stress/UPR. Whether ER stress/UPR contributes RyR1 myopathies arising from RyR1 mutations at other locations in the RyR1 structure also requires further investigation. While more work is needed to confirm that this persistent ER stress is a defining component of the human RyR1 myopathies and to determine if the potential benefits of $4 \mathrm{PBA}$ outweigh any risks in treating this unique subclass of RyR1 myopathy, our work raises the possibility that the clinically approved drug, $4 \mathrm{PBA}$, could be repurposed for use in humans with RyR1 myopathies arising from ER stress.

\section{Methods}

Generation of $\boldsymbol{R y R} \boldsymbol{1}^{\mathbf{1 4 8 9 5 T} / \boldsymbol{W T}}$ knock-in mice. A genomic clone containing exons 14-23 of mice Ryr1 was isolated from a $129 / \mathrm{SvJ} \lambda \mathrm{KO}-1$ library. A $4.9 \mathrm{~Kb}$ SalI-SalI RyR1 fragment was cloned into pBluescript II KS + (pBS, Stratagene) to generate the plasmid pMGC1. The pMGC1 was used for site-directed mutagenesis (QuickChange XL, Stratagene) to introduce both the I4898T (I4895T in mice) mutation and a new BamHI restriction site. The Sal I-Sal I region containing the I4898T mutation was completely sequenced and subcloned back into the RyR1 genomic clone. In the process of screening the $129 / \mathrm{SvJ} \lambda \mathrm{KO}-1$ library, a tetracycline resistance gene (TetR derived from the pKOEZ-50 plasmid provided by $\mathrm{P}$. Zhang) flanked by two NotI restriction sites was introduced into intron 17 of the RyR1 genomic clone. The TetR was then cut out with NotI and replaced with a lox P flanked NeoR gene expressed from the phosphoglycerate kinase promoter (PGK-NeoR) and a TetR cassette to obtain the final targeting vector $\left(\right.$ Ryr1 $\left.1^{I 4898 T ~ N e o}\right)$. The targeting vector was linearized with PmeI and electroporated into AB2.2 129 Sv/J ES cells. DNA was isolated from G418 and gancyclovir double resistant clones and subjected to Southern blot analysis. Correctly targeted ES cells were further verified for the presence of the mutation by PCR followed by restriction digestion with BamHI and direct sequencing. One correctly targeted ES clone carrying the I4898T mutation was injected into C57BL/6 blastocysts, and resulting chimeras were mated with $\mathrm{C} 57 \mathrm{BL} / 6$ mice. The clone gave rise to germline transmission resulting F1 Ryr1 I4895Tneo/ + mice. The Ryr1 ${ }^{\text {I4898Tneo/ }+}$ mice were mated onto Tg (EIIA-Cre) mice (a gift from Dr Graeme Mardon, Baylor College of Medicine, Houston, TX) to remove the floxed PGK-NeoR/TetR cassette, thus generating heterozygous (Ht) RyrI I4898T/wt mice. RyrI ${ }^{14898 T / w t}$ mice were backcrossed on C57BL/6 background. Experimental protocols for animal research were approved by Institutional Animal Care and Use Committees (IACUC) at Baylor College of Medicine (animal protocol AN2656). Male mice were used in the current studies. Ongoing studies are assessing the phenotype and effect of 4PBA in the female mice. Generation of the IT mice is outlined in Supplementary Fig. 1.

Materials. N-benzyl-p-toluene sulfonamide (BTS) was purchased from Tocris Biosciences and 4-chloro-m-cresol (4CmC) from PFALTZ \& BAUER INC. The calcium dyes fura-2 AM and mag-fluo4 AM and the mitochondrial ROS sensor, MitoSOX Red were purchased from ThermoFisher. Xestospongin $\mathrm{C}$ was obtained from Abcam. RU-360 was purchased from Calbiochem. 4-phenylbutyric acid (4PBA) and all other chemicals were purchased from Sigma-Aldrich unless otherwise stated. Acrylamide solution (30\%), protease inhibitor cocktail and molecular weight marker for western blotting were purchased from GenDEPOT. All antibodies used are listed in Supplementary Table 1.

Fibretyping with cryosections and immunostaining. Muscles (soleus, EDL and diaphragm) were dissected, embedded in OCT compound (Tissue-Tek Cryomold (SAKURA)), and frozen in precooled 2-methylbutane. Frozen muscles were sectioned at $9 \mu \mathrm{m}$ thickness using a cryostat microtome (SHANDON Cryotome E, Thermo Electron Corporation). For fibre type staining, sections were rehydrated with PBS for $10 \mathrm{~min}$ and incubated at $4{ }^{\circ} \mathrm{C}$ overnight with anti-MHCI (DSHB, BA-F8, IgG 2 b, 1:50 dilution) and anti-MHCIIa (DSHB, SC-71, IgG 1 , 1:50 dilution) antibodies (see Supplementary Table 1 for details) ${ }^{56}$. Sections were washed twice in PBS and incubated at room temperature for $90 \mathrm{~min}$ AlexaFluor594-conjugated goat anti-mouse IgG $_{2 b}$ and AlexaFluor-488-conjuated goat antimouse IgG $_{1}$ (Life Technologies) secondary antibodies diluted 1:200 in PBS. After washing with PBS, muscle sections were mounted with Fluoromount-G mounting media (SouthernBiotech) supplemented with DAPI for nuclear staining. Imaging was performed under a fluorescence microscope (Olympus). Fibre type number was quantified and normalized by the total number of muscle fibres per field.

Fibre cross-sectional area. Fibre type images were imported into Photoshop CSE v. 10.0 (Adobe Systems, San Jose, CA) for analysis. After establishing the measurement scale by tracing a scale bar in each image $(\mu \mathrm{m})$ CSA of muscle fibres was measured by tracing the external border of individual muscle fibre using the Magnetic Lasso tool ${ }^{56}$. For accurate analysis muscle fibres exhibiting evidence of damage or processing artefacts were excluded from the analysis. Recorded values were exported into a spreadsheet program (Excel, Microsoft Inc.) for analysis and CSA values were described as $\mu \mathrm{m}^{2}$.

Wire hang test. A wire hang test ${ }^{57}$ was employed to assess whole-body muscle strength and endurance. Mice were suspended by the forelimbs at the centre of a metallic wire ( $1 \mathrm{~mm}$ in diameter, $65 \mathrm{~cm}$ in length), which is elevated at $40 \mathrm{~cm}$ above the surface level. As the primary outcome, the holding impulse $\left(g^{*} s\right)$ is calculated as the product of the body mass ( $g$ ) and the longest suspension time (s) during the 3-min test period. These studies were blinded to testers and the mice being analysed were randomized.

Mouse activity. Briefly, to assess the food intake and cage activity, mice were placed in the Comprehensive Laboratory Animal Monitoring System (CLAM System, Columbus Instruments) equipped with running wheel at room temperature and monitored for up to 2 weeks before and after drug treatment ${ }^{58}$. 


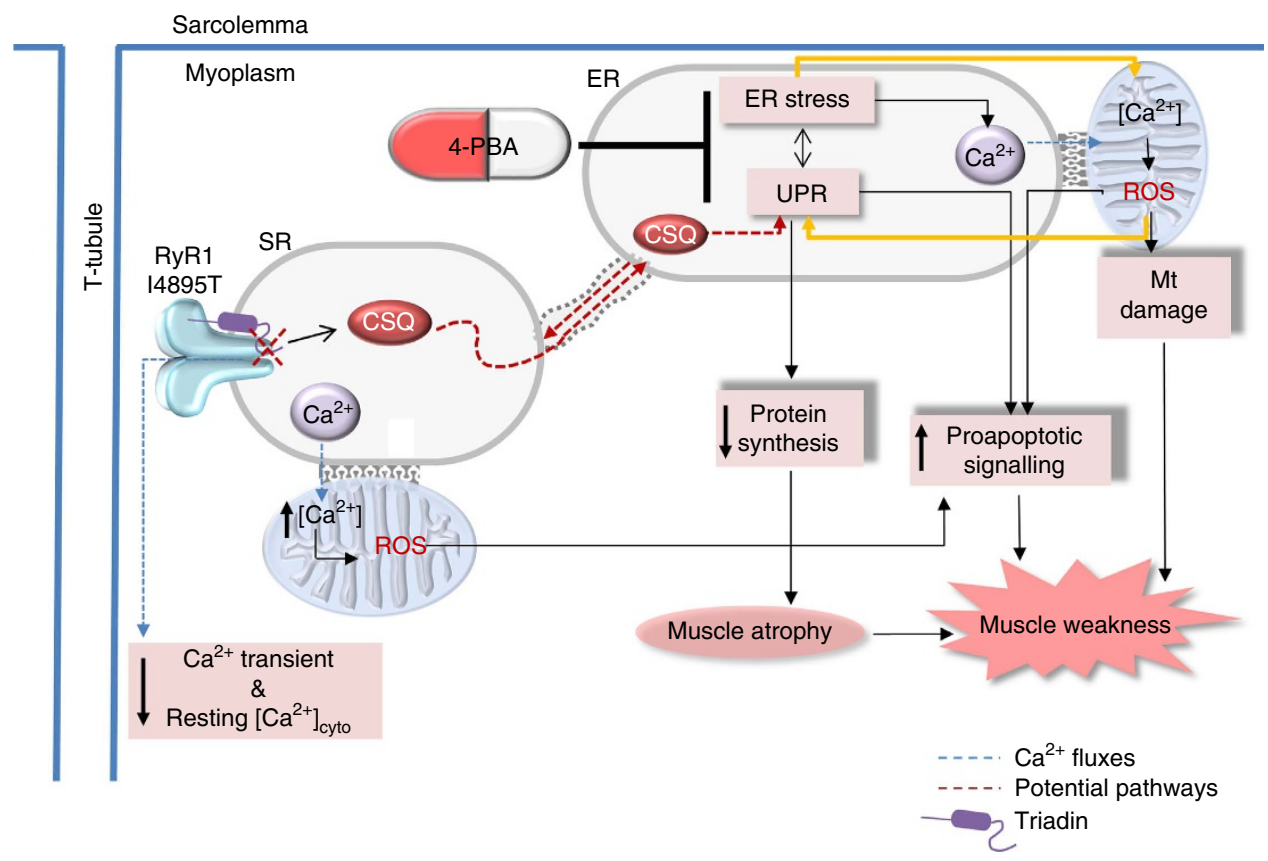

Figure 9 | Model for effects of the IT mutation and 4PBA. The RyR1 IT mutation alters the interactions of RyR1 with SR lumenal proteins to drive ER stress/UPR, leading to decreased protein synthesis, increased mitochondrial $\mathrm{Ca}^{2+}$ uptake and ROS production (which could feed forward to further increase ER stress), mitochondrial damage and activation of proapoptotic pathways. 4PBA reverses pathological signalling pathways and improves muscle functions in IT mice.

Muscle force frequency and fatigue. Strips of diaphragm muscle and intact soleus and EDL muscles were removed and immersed in Kreb's ringer solution $\left(137 \mathrm{mM} \mathrm{NaCl}, 5 \mathrm{mM} \mathrm{KCl}, 1 \mathrm{mM} \mathrm{NaH} \mathrm{PO}_{4}, 24 \mathrm{mM} \mathrm{NaHCO}, 5 \mathrm{mM}\right.$ Glucose, $2 \mathrm{mM} \mathrm{CaCl}_{2}, 1 \mathrm{mM} \mathrm{MgSO}_{4}, \mathrm{pH}$ 7.4) oxygenated with a $95 / 5 \%$ mixture of $\mathrm{O}_{2} / \mathrm{CO}_{2}$ (ref. 56). Muscles were suspended from a force transducer and anchored in a test chamber filled with Kreb's solution. After a 20 -min equilibration period, muscle optimal length $\left(l_{\mathrm{o}}\right)$ and optimal stimulation voltage was determined via single twitch force generation measurements applied using platinum electrodes attached to a Grass S48 stimulator and recorded within Chart5 (version 5.2) software. Force frequency measurements were obtained using frequencies from $1-300 \mathrm{~Hz}$ at $200 \mathrm{~ms} /$ train followed by a fatigue protocol performed over $5 \mathrm{~min}$ per muscle. The specific fatigue protocol for each muscle used was for the soleus: $15 \mathrm{~Hz}, 200 \mathrm{~ms}$ duration, $1 \mathrm{~s}$ intervals) and for EDL: $60 \mathrm{~Hz}, 200 \mathrm{~ms}$ duration, $1 \mathrm{~s}$ intervals. All experiments were performed at $35^{\circ} \mathrm{C}$.

Immunohistochemistry. Intact FDB fibres were plated on extracellular matrix and cultured overnight at $37^{\circ} \mathrm{C}$. Fibres were fixed for $10 \mathrm{~min}$ in PBS containing $4 \%$ formalin and $100 \mu \mathrm{M}$ EGTA and then washed $3 \times 5 \mathrm{~min}$ in PBS. Fibres were then permeabilized for $30 \mathrm{~min}$ in $1 \%$ Triton X-100 and blocked for $1 \mathrm{~h}$ in blocking buffer ( $2 \%$ goat serum, $0.1 \%$ Triton X-100 and $0.5 \%$ BSA). Antibodies to RyR1 (Thermo Scientific MA3-925, 1:300 dilution) and CSQ (Thermo Scientific, PA1-913, 1:400 dilution) were diluted in blocking buffer and applied overnight at $4{ }^{\circ} \mathrm{C}$. After washing $3 \times 5 \mathrm{~min}$ in PBS, fibres were incubated with Alexa Fluor conjugated secondary antibodies for $2 \mathrm{~h}$ at room temperature before being mounted using VECTASHIELD mounting media. Fibres were imaged in the Optical Imaging and Vital Microscopy Core at BCM on a Zeiss LSM 780 confocal microscope using the $\times 60$ oil objective and images were quantified in Image J.

Intracellular $\mathrm{Ca}^{\mathbf{2}+}$ handling. FDB muscles were dissected and fibres were isolated by collagenase digestion ${ }^{47}$. Fibres were imaged within $24 \mathrm{~h}$ of isolation. Fibres were loaded with $5 \mu \mathrm{M}$ Mag-Fluo $4 \mathrm{AM}$ for $30 \mathrm{~min}$ at room temperature followed by 30 min washout with Tyrode's solution containing: $121 \mathrm{mM} \mathrm{NaCl}, 5 \mathrm{mM} \mathrm{KCl}$, $1.8 \mathrm{mM} \mathrm{CaCl}_{2}, 500 \mu \mathrm{M} \mathrm{MgCl}_{2}, 400 \mu \mathrm{M} \mathrm{NaH}_{2} \mathrm{PO}_{4}, 100 \mu \mathrm{M}$ EDTA, $5.5 \mathrm{mM}$ glucose and $24 \mathrm{mM} \mathrm{NaHCO}_{3}, \mathrm{pH}$ regulated with bubbling of $\mathrm{CO}_{2} / \mathrm{O}_{2} 5 / 95 \%$, supplemented with $40 \mu \mathrm{M}$ BTS to inhibit muscle contraction. To assess the frequency dependence of $\mathrm{Ca}^{2+}$ release, electrical stimulation was applied using two platinum electrodes positioned longitudinally either side of the fibre at the following stimulation frequencies: $1 \mathrm{~Hz}, 20 \mathrm{~Hz}, 60 \mathrm{~Hz}, 100 \mathrm{~Hz}(250 \mathrm{~ms}$ train duration), separated by a 1-min rest period. Fibres were imaged using a Zeiss LSM 510 meta confocal microscope in line scan mode with a $\times 20$ objective (EC Plan-Neofluar). One line was acquired every $1.15 \mathrm{~ms}(3.66 \mu \mathrm{s} / \mathrm{pixel} / \mathrm{time})$. Mag-fluo 4 was excited at $488 \mathrm{~nm}$ and emitted light was detected with a $505 \mathrm{~nm}$ long-pass filter. Analyses of the area under the curve and $\mathrm{Ca}^{2+}$ transient kinetics was made using Sigmaplot 13 .
In other experiments Mag Fluo 4 and Fura 2 loaded FDB fibres were imaged with an inverted Nikon Eclipse TE-200 microscope equipped with a Lambda DG5 illumination system and a Rolera MGi-plus CCD camera (Photometrics). Mag Fluo 4 loaded FDB fibres were stimulated with $100 \mathrm{~Hz}$ trains ( $250 \mathrm{~ms}$ duration, every $1.5 \mathrm{~s} ; 0.17$ duty cycle) for $300 \mathrm{~s}$ and Mag Fluo 4 was excited at $500 \mathrm{~nm}$ and emitted light was collected continuously at $>510 \mathrm{~nm}$. To measure baseline cytoplasmic $\mathrm{Ca}^{2+}$ isolated FDB fibres were loaded with $10 \mu \mathrm{M}$ Fura-2AM for $1 \mathrm{~h}$ at room temperature followed by a 30 -min washout with Tyrode's solution. Fibres were excited at $340 / 380 \mathrm{~nm}$ and fluorescence emission was measured at $510 \mathrm{~nm}$. Data were acquired using Metafluor (Version 7.8) and analysed in Sigmaplot 13.

Western blotting. Briefly, muscles were lysed with bead homogenizer (Precelly 24 lysis \& homogenization, Bertin technologies) in ice-cold RIPA buffer:

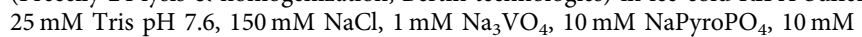
$\beta$-glycerophosphate, $10 \mathrm{mM} \mathrm{NaF}, 1 \mathrm{mM}$ PMSF, $1 \mathrm{X}$ protease inhibitor cocktail (Santa Cruz), 1\% NP40, 1\% sodium deoxycholate and 0.1\% SDS. After quantification by BCA protein assay (Thermo Scientific) equal amounts of muscle homogenates were resolved by SDS-PAGE, transferred to PVDF membrane (Millipore, Billercia, United States) and probed using primary antibodies from the vendors and at the dilutions shown in Supplementary Table 1 with LI-COR IRDye secondary antibodies (LI-COR Inc, Lincoln, United States). Immunoreactive bands were visualized and quantified by densitometry using the Odyssey Infrared Imaging System and software (LI-COR). To control for variations among different western blots and allow for multiple repeats, the absorbance intensity for each band on the western blot was normalized to the absorbance intensity of corresponding band from the control mice from the same western blot, averaged with values obtained from other western blots and plotted as \% control. Some blots had multiple controls and the absorbance of the specific band was normalized to the average ${ }^{56}$. Full length western blots are show in Supplementary Fig. 9.

Transmission electron microscopy for ultrastructure. Electron microscopy was performed by using methods modified from Boncompagni ${ }^{14}$. Muscle segments were postfixed in $1 \% \mathrm{OsO}_{4}$ for $1 \mathrm{~h}$ at $4{ }^{\circ} \mathrm{C}$, en block-stained in saturated aqueous uranyl acetate for $1 \mathrm{~h}$ and embedded in Spurr's Low Viscosity resin. Ultrathin sections $(50-60 \mathrm{~nm})$ were cut with an ultramicrotome Leica U7 (Leica Microsystem, Austria) using a Diatome diamond knife (Diatome Ltd. $\mathrm{CH}-2501$ Biel, Switzerland). Sections were stained in saturated aqueous uranyl acetate and lead citrate solutions. Sections were imaged at $\times 3500$.

TUNEL and caspase activity assay. Diaphragm, EDL and soleus muscle tissues were lysed in lysis buffer (Triton X-100 0.1\%, Tris $\mathrm{HCl} \mathrm{pH} \mathrm{8,5} \mathrm{mM,} \mathrm{EGTA} 20 \mathrm{mM}$, 
EDTA $20 \mathrm{mM}$ ) and homogenized with polytron homogenizer (Precellys 24, Bertin Technologies). Protein concentrations were measured using Lowry assay. Cell apoptosis ELISA analysis was performed according to manufacturer's instructions after appropriate dilutions (Roche). For the TUNEL assay, frozen sections of soleus were fixed with fixation solution (4\% paraformaldehyde in PBS pH 7.4) for $20 \mathrm{~min}$, washed with PBS for $30 \mathrm{~min}$ and then incubated with permeabilization solution $(0.1 \%$ Triton X-100, $0.1 \%$ sodium citrate). Fixed and permeabilized slides were treated with TUNEL reaction mixture (terminal deoxynucleotidyl transferase from calf thymus, recombinant in E. coli and nucleotide mixture - Roche). Nuclear stain DAPI was used to label the nuclei. For caspase activation assay, acetyl-AspGlu-Val-Asp-7-amido-4-methylcoumarin (Ac-DEVD-AMC) was used as substrate. Caspase activity was calculated from changes in fluorescent AMC (Excitation $360 \mathrm{~nm}$, emission $460 \mathrm{~nm}$ )

Oxyblot for oxidized proteins. Oxidized proteins were assessed by the OxyBlot Protein Oxidation Detection Kit (Millipore) in $10 \mu \mathrm{g}$ of soleus, EDL and diaphragm lysates following the manufacturer's protocol.

Mitochondrial and cytosolic fractionation. Fresh muscles were homogenized using a glass homogenizer with ice-cold mitochondrial isolation buffer $(10 \mathrm{mM}$ Tris- $\mathrm{HCl} \mathrm{pH} \mathrm{7.2,} 320 \mathrm{mM}$ sucrose, $1 \mathrm{mM}$ ethylene diamine tetra acetic acid, $1 \mathrm{mM}$ dithiothreitol, $1 \mathrm{mg} \mathrm{ml}^{-1}$ bovine serum albumin) and centrifuged at $1,500 \mathrm{~g}$ for $5 \mathrm{~min}$ to remove debris and nuclear fractions. Supernatant was transferred to a new tube and was further centrifuged at $15,000 \mathrm{~g}$ for $20 \mathrm{~min}$ for the phase separation of the mitochondrial pellet and cytosolic supernatant. Mitochondrial pellet was lysed in RIPA buffer $\left(25 \mathrm{mM}\right.$ Tris pH 7.6, $150 \mathrm{mM} \mathrm{NaCl}, 1 \mathrm{mM} \mathrm{Na}_{3} \mathrm{VO}_{4}, 10 \mathrm{mM}$ $\mathrm{NaPyroPO}_{4}, 10 \mathrm{mM} \beta$-glycerophosphate, $10 \mathrm{mM} \mathrm{NaF}, 1 \mathrm{mM}$ PMSF, $1 \mathrm{X}$ protease inhibitor cocktail (Santa Cruz), 1\% NP40, 1\% sodium deoxycholate, and $0.1 \%$ SDS (reagents from Sigma Aldrich) and protein concentrations of each fraction were determined by the BCA Protein Assay (Thermo Scientific), according to the manufacturer's protocol.

XBP1 splicing. Total RNA was extracted from mouse tissue using TRIzol reagent (Life Technologies), and reverse transcribed into cDNA using iScript cDNA Synthesis Kit (BIO-RAD). Quantitative real-time polymerase chain reaction (qRT-PCR) was performed with iQ SYBR Green Supermix (BIO-RAD) using primer sets specific for target and reference genes (PrimerBank, MGH). The relative splicing level of Xbp1 was determined by normalizing the expression of spliced $\mathrm{Xbpl}(\mathrm{S})$ to unspliced $\mathrm{Xbpl}(\mathrm{U})$ mRNA. The relative transcription levels of all other genes were determined by normalizing the expression of target genes to the reference $18 \mathrm{~s}$ rRNA. Relative gene expression was calculated based on the reaction cycle threshold $(\mathrm{Ct})$ values using Pfaff method ${ }^{59}$. Primers: Xbpls forward $5^{\prime}$-GAGTCCGCAGCAGGTG- ${ }^{\prime}$ and reverse $5^{\prime}$-GTGTCAGAGTCCATGGGA-3'; Xbplu forward $5^{\prime}$-GACTATGTGCACCTCTGCAG-3' and reverse $5^{\prime}$-CTGGGA GTTCCTCCAGACTA-3'.

Mitochondrial ROS. Mitochondrial ROS production was measured using methods modified from Cheng et al. ${ }^{60}$ and Aydin et al. ${ }^{61}$. Briefly, single FDB fibres were incubated at room temperature with $5 \mu \mathrm{M}$ MitoSOX Red in Tyrodes solution for $15 \mathrm{~min}$, followed by a 45 -min washout period in indicator-free Tyrodes solution. MitoSOX loaded fibres were imaged on a Zeiss LSM 510 meta laser scanning confocal microscope using a $\times 20$ objective. Fibres were excited with an argon laser at $488 \mathrm{~nm}$ and emission was collected using a 560LP filter. XY images of baseline mitoSOX fluorescence were always captured within $20 \mathrm{~min}$ of completion of mitoSOX loading. Antimycin A $(20 \mu \mathrm{M})$ was added to the chamber at the end of each experiment to verify the rise in MitoSOX fluorescence was mitochondrial. To determine the effect of blocking mitochondrial $\mathrm{Ca}^{2+}$ uptake or $\mathrm{IP}_{3} \mathrm{R}$ mediated $\mathrm{Ca}^{2+}$ release on mitochondrial ROS production, in some experiments fibres were incubated for $1 \mathrm{~h}$ with $10 \mu \mathrm{M}$ RU-360, or with $1 \mu \mathrm{M}$ Xestospongin $\mathrm{C}$ for $20 \mathrm{~min}$ before MitoSOX loading.

SUnSET assay for detection of puromycin-labelled proteins. For assessment of newly synthesized proteins we used in vivo puromycin labelling ${ }^{46,62}$. Briefly, mice (14-15 weeks of age) were deprived of food for $6 \mathrm{~h}$ and refed for $2 \mathrm{~h}$. Propofol $\left(18 \mu \mathrm{g} \mathrm{g}^{-1}\right)$ was intraperitoneally injected in mice after $70 \mathrm{~min}$ of refeeding. After $10 \mathrm{~min}$, mice were intraperitoneally injected with puromycin $\left(0.04 \mu \mathrm{mol} \mathrm{g}^{-1}\right.$ body weight) and killed $35 \mathrm{~min}$ later. Excised muscles were processed for western blotting with anti-puromycin antibody (KeraFAST EQ-001, 1:1,000 dilution). Puromycin-labelled proteins were normalized to total proteins of the same blot stained with the Swift Membrane Stain kit (G-Biosciences).

Chronic treatment with 4BPA. Age-matched IT and WT littermates were used for chronic (up to 6 weeks) treatment. For chronic treatment, 4PBA (Sigma) was dissolved at a concentration of $200 \mathrm{mg} \mathrm{dl}^{-1}$ and treated ad-libitum. Approximate water consumption was 3-5 ml per day per mouse. Therefore, each mouse consumes about 6-10 mg per day per mouse.
Aggresome detection. All procedures were performed as described in the manufacturer's protocol (Enzo Life Sciences). Briefly, muscle frozen sections from soleus and EDL were placed at room temperature for $10 \mathrm{~min}$ and rehydrated in 1X PBS. After removing excess buffer dual detection reagent $(1 \mu$ l of ProteoStat Aggresome detection reagent and $2 \mu \mathrm{l}$ of Hoechst 33342 in $200 \mu \mathrm{l}$ of $1 \mathrm{X}$ assay buffer) was added to sections and incubated for $2 \mathrm{~h}$ at room temperature. After washing with $1 \mathrm{X}$ PBS, slides were mounted with coverslip using Fluoromount-G (SouthernBiotech). Imaging was performed under $\times 20$ magnification through an Olympus DP70 camera (Olympus America, Center Valley, PA). Fluorescence intensity of each image was quantified using Image-J software.

Statistical analyses. Differences between means of two groups were analysed for significance using two tailed Student's $t$-tests. Data are also subjected to a normality test. If data fail the normality test they are then subjected to Mann-Whitney rank sum test. Only those data with ${ }^{\star} P<0.05,{ }^{* *} P<0.01$ or ${ }^{* *} P<0.001$ are considered significant. Outliers, defined as those values that were greater than 2 standard deviations away from the mean, were removed.

Data availability. The data that support the findings of this study are available from the corresponding author upon reasonable request.

\section{References}

1. Maggi, L. et al. Congenital myopathies - clinical features and frequency of individual subtypes diagnosed over a 5-year period in the United Kingdom. Neuromuscul. Disord. 23, 195-205 (2013).

2. Lillis, S., Abbs, S., Mueller, C. R., Muntoni, F. \& Jungbluth, H. Clinical utility gene card for: Central core disease. Eur. J. Hum. Genet. 20 http://dx.doi.org/ 10.1038/ejhg.2011.179 (2012).

3. Avila, G. \& Dirksen, R. T. Functional effects of central core disease mutations in the cytoplasmic region of the skeletal muscle ryanodine receptor. J. Gen. Physiol. 118, 277-290 (2001).

4. Loy, R. E. et al. Muscle weakness in Ryr1I4895T/WT knock-in mice as a result of reduced ryanodine receptor $\mathrm{Ca} 2+$ ion permeation and release from the sarcoplasmic reticulum. J. Gen. Physiol. 137, 43-57 (2011).

5. Durham, W. J. et al. RyR1 S-nitrosylation underlies environmental heat stroke and sudden death in Y522S RyR1 knockin mice. Cell 133, 53-65 (2008).

6. Andersson, D. C. \& Marks, A. R. Fixing ryanodine receptor Ca leak - a novel therapeutic strategy for contractile failure in heart and skeletal muscle. Drug. Discov. Today Dis. Mech. 7, e151-e157 (2010).

7. Lanner, J. T. et al. AICAR prevents heat-induced sudden death in RyR1 mutant mice independent of AMPK activation. Nat. Med. 18, 244-251 (2012).

8. Lynch, P. J. et al. A mutation in the transmembrane/luminal domain of the ryanodine receptor is associated with abnormal $\mathrm{Ca} 2+$ release channel function and severe central core disease. Proc. Natl Acad. Sci. USA 96, 4164-4169 (1999).

9. Davis, M. R. et al. Principal mutation hotspot for central core disease and related myopathies in the C-terminal transmembrane region of the RYR1 gene. Neuromuscul. Disord. 13, 151-157 (2003).

10. Tilgen, N. et al. Identification of four novel mutations in the C-terminal membrane spanning domain of the ryanodine receptor 1 : association with central core disease and alteration of calcium homeostasis. Hum. Mol. Genet. 10, 2879-2887 (2001).

11. Shuaib, A., Paasuke, R. T. \& Brownell, K. W. Central core disease. Clinical features in 13 patients. Medicine 66, 389-396 (1987).

12. Hernandez-Lain, A. et al. de novo RYR1 heterozygous mutation (I4898T) causing lethal core-rod myopathy in twins. Eur. J. Med. Genet. 54, 29-33 (2011).

13. Zvaritch, E. et al. An Ryr1I4895T mutation abolishes Ca2 + release channel function and delays development in homozygous offspring of a mutant mouse line. Proc. Natl Acad. Sci. USA 104, 18537-18542 (2007).

14. Boncompagni, S., Loy, R. E., Dirksen, R. T. \& Franzini-Armstrong, C. The I4895T mutation in the type 1 ryanodine receptor induces fiber-type specific alterations in skeletal muscle that mimic premature aging. Aging Cell 9, 958-970 (2010).

15. Jungbluth, H. \& Voermans, N. C. Congenital myopathies: not only a paediatric topic. Curr. Opin. Neurol. 29, 642-650 (2016).

16. Eisner, V., Csordás, G. \& Hajnóczky, G. Interactions between sarcoendoplasmic reticulum and mitochondria in cardiac and skeletal muscle pivotal roles in $\mathrm{Ca} 2+$ and reactive oxygen species signaling. J. Cell Sci. 126, 2965-2978 (2013).

17. Kaufman, R. J. \& Malhotra, J. D. Calcium trafficking integrates endoplasmic reticulum function with mitochondrial bioenergetics. Biochim. Biophys. Acta (BBA)-Mol. Cell Res. 1843, 2233-2239 (2014).

18. Boncompagni, S. et al. Mitochondria are linked to calcium stores in striated muscle by developmentally regulated tethering structures. Mol. Biol. Cell 20, 1058-1067 (2009). 
19. Hajnoczky, G., Robb-Gaspers, L. D., Seitz, M. B. \& Thomas, A. P. Decoding of cytosolic calcium oscillations in the mitochondria. Cell 82, 415-424 (1995).

20. Belmonte, S. \& Morad, M. 'Pressure-flow'-triggered intracellular Ca2 + transients in rat cardiac myocytes: possible mechanisms and role of mitochondria. J. Physiol. 586, 1379-1397 (2008).

21. Mironov, S. L., Ivannikov, M. V. \& Johansson, M. $\left[\mathrm{Ca}^{2+}\right] i$ signaling between mitochondria and endoplasmic reticulum in neurons is regulated by microtubules. From mitochondrial permeability transition pore to $\mathrm{Ca} 2+-$ induced Ca2 + release. J. Biol. Chem. 280, 715-721 (2005).

22. Brookes, P. S., Yoon, Y., Robotham, J. L., Anders, M. W. \& Sheu, S. S. Calcium, ATP, and ROS: a mitochondrial love-hate triangle. Am. J. Physiol. Cell Physiol. 287, C817-C833 (2004).

23. Csordas, G. \& Hajnoczky, G. SR/ER-mitochondrial local communication: calcium and ROS. Biochim. Biophys. Acta 1787, 1352-1362 (2009).

24. Robinson, K. M. et al. Selective fluorescent imaging of superoxide in vivo using ethidium-based probes. Proc. Natl Acad. Sci. USA 103, 15038-15043 (2006).

25. Casas, M. et al. IP(3)-dependent, post-tetanic calcium transients induced by electrostimulation of adult skeletal muscle fibers. J. Gen. Physiol. 136, 455-467 (2010).

26. Ainbinder, A., Boncompagni, S., Protasi, F. \& Dirksen, R. T. Role of mitofusin-2 in mitochondrial localization and calcium uptake in skeletal muscle. Cell Calcium 57, 14-24 (2015).

27. Rossi, A. E., Boncompagni, S., Wei, L., Protasi, F. \& Dirksen, R. T. Differential impact of mitochondrial positioning on mitochondrial $\mathrm{Ca}(2+)$ uptake and $\mathrm{Ca}(2+)$ spark suppression in skeletal muscle. Am. J. Physiol. Cell Physiol. 301, C1128-C1139 (2011).

28. Dirksen, R. T. Sarcoplasmic reticulum-mitochondrial through-space coupling in skeletal muscle. Appl. Physiol. Nutr. Metab. 34, 389-395 (2009).

29. Ross, C. A. et al. Inositol 1,4,5-trisphosphate receptor localized to endoplasmic reticulum in cerebellar Purkinje neurons. Nature 339, 468-470 (1989).

30. Goonasekera, S. A. et al. Triadin binding to the C-terminal luminal loop of the ryanodine receptor is important for skeletal muscle excitation-contraction coupling. J. Gen. Physiol. 130, 365-378 (2007).

31. Lee, J. M. et al. Negatively charged amino acids within the intraluminal loop of ryanodine receptor are involved in the interaction with triadin. J. Biol. Chem. 279, 6994-7000 (2004).

32. Marty, I. Triadin regulation of the ryanodine receptor complex. J. Physiol. 593, 3261-3266 (2015).

33. Wei, L., Gallant, E. M., Dulhunty, A. F. \& Beard, N. A. Junctin and triadin each activate skeletal ryanodine receptors but junctin alone mediates functional interactions with calsequestrin. Int. J. Biochem. Cell Biol. 41, 2214-2224 (2009).

34. Malhotra, J. D. \& Kaufman, R. J. ER stress and its functional link to mitochondria: role in cell survival and death. Cold Spring Harb. Perspect. Biol. 3, a004424 (2011)

35. Blackshaw, S. et al. Type 3 inositol 1,4,5-trisphosphate receptor modulates cell death. FASEB J. 14, 1375-1379 (2000).

36. Raturi, A. \& Simmen, T. Where the endoplasmic reticulum and the mitochondrion tie the knot: the mitochondria-associated membrane (MAM). Biochim. Biophys. Acta 1833, 213-224 (2013).

37. Ezerman, E. B. \& Ishikawa, H. Differentiation of the sarcoplasmic reticulum and $\mathrm{T}$ system in developing chick skeletal muscle in vitro. J. Cell Biol. 35, 405-420 (1967).

38. Chaudhari, N., Talwar, P., Parimisetty, A., Lefebvre d'Hellencourt, C. \& Ravanan, P. A molecular web: endoplasmic reticulum stress, inflammation, and oxidative stress. Front. Cell. Neurosci. 8, 213 (2014).

39. van Vliet, A. R., Verfaillie, T. \& Agostinis, P. New functions of mitochondria associated membranes in cellular signaling. Biochim. Biophys. Acta (BBA)Mol. Cell Res. 1843, 2253-2262 (2014)

40. van Vliet, A. R., Verfaillie, T. \& Agostinis, P. New functions of mitochondria associated membranes in cellular signaling. Biochim. Biophys. Acta 1843, 2253-2262 (2014).

41. Valle, G., Boncompagni, S., Sacchetto, R., Protasi, F. \& Volpe, P. Post-natal heart adaptation in a knock-in mouse model of calsequestrin 2-linked recessive catecholaminergic polymorphic ventricular tachycardia. Exp. Cell Res. 321, 178-189 (2014)

42. Cai, W.-F. et al. Ablation of junctin or triadin is associated with increased cardiac injury following ischaemia/reperfusion. Cardiovasc. Res. 94, 333-341 (2012).

43. Sano, R. \& Reed, J. C. ER stress-induced cell death mechanisms. Biochim. Biophys. Acta 1833, 3460-3470 (2013).

44. Pinton, P., Giorgi, C., Siviero, R., Zecchini, E. \& Rizzuto, R. Calcium and apoptosis: ER-mitochondria $\mathrm{Ca} 2+$ transfer in the control of apoptosis. Oncogene 27, 6407-6418 (2008).
45. Goodman, C. A. \& Hornberger, T. A. Measuring protein synthesis with SUnSET: a valid alternative to traditional techniques? Exerc. Sport Sci. Rev. 41, 107-115 (2013).

46. Schmidt, E. K., Clavarino, G., Ceppi, M. \& Pierre, P. SUnSET, a nonradioactive method to monitor protein synthesis. Nat. Methods 6, 275-277 (2009).

47. Perlmutter, D. H. Chemical chaperones: a pharmacological strategy for disorders of protein folding and trafficking. Pediatr. Res. 52, 832-836 (2002).

48. Csordas, G. et al. Structural and functional features and significance of the physical linkage between ER and mitochondria. J. Cell Biol. 174, 915-921 (2006).

49. Green, D. R. \& Kroemer, G. The pathophysiology of mitochondrial cell death Science 305, 626-629 (2004).

50. Bernardi, P. Mitochondrial transport of cations: channels, exchangers, and permeability transition. Physiol. Rev. 79, 1127-1155 (1999).

51. Wilmshurst, J. M. et al. RYR1 mutations are a common cause of congenital myopathies with central nuclei. Ann. Neurol. 68, 717-726 (2010).

52. Clarke, N. F. et al. Recessive mutations in RYR1 are a common cause of congenital fiber type disproportion. Hum. Mutat. 31, E1544-E1550 (2010).

53. Dibble, C. C. \& Manning, B. D. Signal integration by mTORC1 coordinates nutrient input with biosynthetic output. Nat. Cell Biol. 15, 555-564 (2013).

54. Qi, X., Hosoi, T., Okuma, Y., Kaneko, M. \& Nomura, Y. Sodium 4phenylbutyrate protects against cerebral ischemic injury. Mol. Pharmacol. 66, 899-908 (2004)

55. Zhou, W. et al. Phenylbutyrate up-regulates the DJ-1 protein and protects neurons in cell culture and in animal models of Parkinson disease. J. Biol. Chem. 286, 14941-14951 (2011).

56. Lee, C. S. et al. $\mathrm{Ca}(2+)$ permeation and/or binding to CaV1.1 fine-tunes skeletal muscle $\mathrm{Ca}(2+)$ signaling to sustain muscle function. Skelet. Muscle 5, 4 (2015).

57. Aartsma-Rus, A. \& van Putten, M. Assessing functional performance in the Mdx mouse model. J. Vis. Exp. 27, e51303 (2014).

58. Georgiou, D. K. et al. Ca2 + binding/permeation via calcium channel, CaV1.1, regulates the intracellular distribution of the fatty acid transport protein, CD36, and fatty acid metabolism. J. Biol. Chem. 290, 23751-23765 (2015).

59. Pfaffl, M. W., Lange, I. G., Daxenberger, A. \& Meyer, H. H. Tissue-specific expression pattern of estrogen receptors (ER): quantification of ER alpha and ER beta mRNA with real-time RT-PCR. Acta Pathol. Microbiol. Immunol. Scand. 109, 345-355, 2001).

60. Cheng, A. J., Bruton, J. D., Lanner, J. T. \& Westerblad, H. Antioxidant treatments do not improve force recovery after fatiguing stimulation of mouse skeletal muscle fibres. J. Physiol. 593, 457-472 (2015).

61. Aydin, J. et al. Increased mitochondrial $\mathrm{Ca} 2+$ and decreased sarcoplasmic reticulum $\mathrm{Ca} 2+$ in mitochondrial myopathy. Hum. Mol. Genet. 18, 278-288 (2009).

62. Lee, C. S. et al. Ligands for FKBP12 increase Ca2 + influx and protein synthesis to improve skeletal muscle function. J. Biol. Chem. 289, 25556-25570 (2014).

\section{Acknowledgements}

Research reported in this publication was supported by the National Institute of Arthritis and Musculoskeletal and Skin Diseases of the National Institutes of Health under Award Number 2R01AR053349-11 to S.L.H. and National Institute of Aging under award number to 1R01AG047924 to E.S. This project was also supported by the Mouse Phenotyping Core at Baylor College of Medicine with funding from the NIH (U54 HG006348) and by the Muscular Dystrophy Association. The EM studies were performed in the BCM Integrated Microscopy Core Facility and the confocal imaging was performed in the BCM Optical Imaging and Vital Microscopy Core Facility. The content is solely the responsibility of the authors and does not necessarily represent the official views of the National Institutes of Health.

\section{Author contributions}

C.S.L., A.D.H. and S.L.H. designed the research, analysed the data and wrote the paper C.S.L., A.D.H., H.W., A.D.-A., A.D.J., M.K., X.X., D.K.G., J.X., C.L., H.A., C.R., K.D. and J.C.M. performed experiments and edited/critiqued the manuscript; W.R.L., G.G.R., E.S., C.S. helped design and supervise specific experiments related to their areas of expertise and edited/critiqued the manuscript.

\section{Additional information}

Supplementary Information accompanies this paper at http://www.nature.com/ naturecommunications

Competing financial interests: The authors declare no competing financial interests. 
Reprints and permission information is available online at http://npg.nature.com/ reprintsandpermissions/

How to cite this article: Lee, C. S. et al. A chemical chaperone improves muscle function in mice with a RyR1 mutation. Nat. Commun. 8, 14659 doi: 10.1038/ncomms14659 (2017).

Publisher's note: Springer Nature remains neutral with regard to jurisdictional claims in published maps and institutional affiliations. (c) (i) This work is licensed under a Creative Commons Attribution 4.0 International License. The images or other third party material in this article are included in the article's Creative Commons license, unless indicated otherwise in the credit line; if the material is not included under the Creative Commons license, users will need to obtain permission from the license holder to reproduce the material. To view a copy of this license, visit http://creativecommons.org/licenses/by/4.0/

(C) The Author(s) 2017 